\title{
Hydrogen bond donor functionalized dioxido- molybdenum(VI) complexes as robust and highly efficient precatalysts for alkene epoxidation
}

Niklas Zwettler, ${ }^{\dagger}$ Jörg A. Schachner, ${ }^{\dagger}$ Ferdinand Belaj ${ }^{\dagger}$ and Nadia C. Mösch-Zanetti ${ }^{\dagger} * *$

$\dagger$ Institute of Chemistry, Inorganic Chemistry, University of Graz, Schubertstrasse 1, 8010 Graz, Austria 


\begin{abstract}
The synthesis of four novel, tridentate aminophenolate ligands HL1-HL4, bearing amide functionalities is reported. Reaction of these ligands with a dioxido molybdenum(VI) precursor led, depending on the choice of solvent, to mononuclear complexes of the type $\left[\mathrm{MoO}_{2} \mathbf{L}(\mathrm{OMe})\right](\mathbf{2}, \mathbf{4}, \mathbf{6})$ or dinuclear complexes $\left[\left\{\mathrm{MoO}_{2} \mathbf{L}\right\}_{2}(\mu-\mathrm{O})\right](\mathbf{1}, \mathbf{3}, \mathbf{5}, \mathbf{7})$, containing one facially, tridentate ONO-ligand per metal center. This synthetic discrimination between dinuclear and mononuclear complexes allows for a comparison between structures and reactivity. Complexes 1-7 were found to be highly active catalysts in the epoxidation of several internal and terminal alkenes. With tert-butyl hydroperoxide (TBHP) as oxidant, precatalyst loadings of $0.0005 \mathrm{~mol} \%$ (5 ppm) could be realized leading to turnover numbers of up to 110000 . The precatalysts also allowed for the use of hydrogen peroxide $(0.1 \mathrm{~mol} \%$ precatalyst $)$ as oxidant as well as various alcohols as "green" solvents, such as ethanol or even tert-butanol (usually an inhibitor of epoxidation).
\end{abstract}

hydrogen bonds $\bullet$ molybdenum $\bullet$ epoxidation $\bullet$ aminoamides $\bullet$ homogeneous catalysis

\title{
1. Introduction
}

The relative ease of ring-opening reactions, and thus the broad variety of possible follow-up products, makes epoxides very important intermediates in chemical synthesis. $[1,2,3,4]$ One of the main synthetic route to epoxides is the oxidation of olefins. Different approaches exist for such a transformation, including the use of stoichiometric amounts of organic peracids as well as hetero- and homogeneous catalytic approaches. [4,5] Homogeneous catalytic protocols usually allow for mild conditions and often employ high valent metal-oxido complexes as precatalysts.[3,6] In the industrial production of propylene oxide via the Arco/Halcon process, such a protocol is realized with molybdenum and tungsten complexes as well as alkyl 
hydroperoxides as oxidants.[1,7] With the prospect to develop environmentally more friendly processes, the use of non-toxic, earth-abundant metals such as molybdenum is of key interest.[8] Especially mononuclear molybdenum(VI) dioxido complexes have proven to be highly active and selective precatalysts for the epoxidation of internal aliphatic olefins, $[9,10,11]$ recently the substrate scope could to some extent be expanded to terminal olefins.[12,13] Furthermore, the use of environmentally benign solvents as well as oxidants remains a major objective.[14] Initially, our research interest in high valent molybdenum compounds focused on oxygen atom transfer as well as the activation of molecular oxygen.[15] We then started to examine the catalytic behavior of the involved molybdenum complexes in the oxidation of olefins. In an endeavor to develop "greener" and more efficient catalytic protocols, our group tested several mono- and dinuclear dioxidomolybdenum(VI), and subsequently also mononuclear oxidorhenium(V) complexes for their capability of catalytic epoxidation of olefins as well as catalytic aerobic oxidation reactions. Whereas the investigated oxidorhenium(V) complexes were robust and in part allowed for the use of hydrogen peroxide as oxidant, they were limited to cyclooctene as substrate.[16] Dioxidomolybdenum systems on the other hand were able to catalyze the oxidation of a broad scope of substrates, albeit with varying selectivities.[9,11,17,18] Especially the use of bidentate Schiff-base ligands with donor sites led to very selective precatalysts.[19] Inspired by the work of Borovik and coworkers,[20] we expanded our library of iminophenolate ligands by introducing amide functionalities as hydrogen bond donors and investigated their coordination chemistry with the dioxidomolybdenum(VI) fragment, finding unusual $\mathrm{C}-\mathrm{C}$ and $\mathrm{C}-\mathrm{N}$ coupling reactions at the metal center.[21] To inhibit such bond formation reactions but retain the structure of the ligands, we decided to investigate the derived aminophenolate derivatives, accessible via reduction of the imine $\mathrm{C}=\mathrm{N}$ bond.[18] 
Herein we present the synthesis of four aminophenolate ligands featuring amide substituents with varying $\mathrm{NH}$ acidity by hydrogenation of the respective iminophenolates with $\mathrm{Pd} / \mathrm{C}-\mathrm{H}_{2}$ or $\mathrm{NaBH}_{4}$. The higher flexibility of those aminophenolate ligands leads to a monoanionic tridentate facial ONO coordination mode resulting in mono- and dinuclear dioxidomolybdenum(VI) complexes of the general formulas $\left[\left\{\mathrm{MoO}_{2} \mathbf{L}\right\}_{2}(\mu-\mathrm{O})\right]$ and $\left[\mathrm{MoO}_{2} \mathbf{L}(\mathrm{OMe})\right]$. The dinuclear complexes are structurally closely related to previously reported complexes, with the additional amide functionalities being of crucial difference.[18] A further important aspect of the system disclosed herein is the fact that both mono- and dinuclear complexes are formed depending on the reaction conditions allowing for a direct comparison of their reactivity. The complexes were found to exhibit exceptionally high activity and functional group tolerance in the catalytic epoxidation of various alkene substrates with TBHP. This is in stark contrast to previously reported structural analogs[18] surpassing the activity of homogeneous epoxidation precatalysts known to date without additional hydrogen bond donors.[9,12,22] In addition we disclose herein the catalytic activity using $\mathrm{H}_{2} \mathrm{O}_{2}$ as oxidant as well as the use of environmentally benign alcoholic solvent.[23]

\section{Experimental Section}

General. Unless specified otherwise, experiments were performed under inert conditions using standard Schlenk equipment. Commercially available chemicals were purchased from Sigma-Aldrich and used as received. No further purification or drying operations have been performed. The metal precursor $\left[\mathrm{MoO}_{2}(\mathrm{acac})_{2}\right][24]$ was synthesized according to known procedures. The used primary amines 2-amino- $N$-(tert-butyl)acetamide and 2-amino- $N$ phenylacetamide were synthesized according to a previously disclosed procedure.[21] Solvents were purified via a Pure-Solv MD-4-EN solvent purification system from Innovative Technology, Inc. Methanol was refluxed over activated magnesium for at least $24 \mathrm{~h}$ and then distilled prior to use. The ${ }^{1} \mathrm{H},{ }^{13} \mathrm{C}$ and HSQC NMR spectra were recorded on a Bruker Optics instrument at 300/75 MHz. Peaks are denoted as singlet (s), broad singlet (bs),doublet 
(d), doublet of doublets (dd), triplet (t), pseudo-doublet (“d"), pseudo-triplet ("t") and multiplet (m). Used solvents and peak assignment are mentioned at the specific data sets. HR-MS $\left(\mathrm{ESI}^{+}\right)$measurements were performed at the University of Graz, Department of Analytical Chemistry using a Thermo Scientific Q-Exactive mass spectrometer in positive ion mode, the used solvent was acetonitrile. Peaks are denoted as cationic mass peaks, and the unit is the according ions mass/charge ratio. For dinuclear compounds the denoted mass peak corresponds to the highest found intensity, full calculated and found isotopic patterns are provided within the supporting information. Gas chromatography mass spectroscopy (GC-MS) measurements have been performed with an Agilent 7890A gas chromatograph (column type Agilent 19091J-433), coupled to an Agilent 5975C mass spectrometer. Samples for infrared spectroscopy were measured on a Bruker Optics ALPHA FT-IR Spectrometer. IR bands are reported with wavenumber $\left(\mathrm{cm}^{-1}\right)$ and intensities (s, strong; m, medium; w, weak). All elemental analyses were measured at the Technical University of Graz, Institute of Inorganic Chemistry using a Heraeus Vario Elementar automatic analyzer.

Catalytic Epoxidation. A Heidolph Parallel Synthesizer 1 was used for all experiments. In a typical epoxidation experiment an aliquot of a stock solution of the respective precatalyst was stirred in $0.5 \mathrm{~mL}$ of the respective solvent and substrate was added. Mesitylene was used as internal standard. After the experiment temperature was reached the respective oxidant was added to start the reaction and samples for GC-MS measurements were withdrawn at given time intervals. GC samples were quenched with $\mathrm{MnO}_{2}$, diluted with ethyl acetate and measured. Gas chromatography mass spectroscopy (GC-MS) measurements have been performed with an Agilent 7890 A gas chromatograph (column type, Agilent 19091J-433), coupled to an Agilent 5975 C mass spectrometer. All yields obtained by GC have an esd of $\pm 5 \%$.

X-ray Diffraction Analyses. Single-crystal X-ray diffraction analyses were measured on a BRUKER-AXS SMART APEX II diffractometer equipped with a CCD detector. All measurements were performed using monochromatized Mo $\mathrm{K}_{\alpha}$ radiation from an Incoatec microfocus sealed tube at $100 \mathrm{~K}$ (cf. Table S1). Absorption corrections were performed semi-empirical from equivalents. Structures were solved by direct methods (SHELXS97)[25] and refined by full-matrix least-squares techniques against $F^{2}$ (SHELXL-2014/6).[25] CCDC 15329781532983 contain the supplementary crystallographic data for this paper. These data can be obtained free of charge from The Cambridge Crystallographic Data Centre via www.ccdc.cam.ac.uk/data_request/cif. Full experimental details for single-crystal X-ray diffraction analyses of all compounds are provided in the SI. 
Ligand Synthesis. All ligands are stable towards air and moisture. They can be stored at ambient conditions for several weeks without decomposition.

Synthesis of $N$-(tert-butyl)-2-((2-hydroxybenzyl)amino)acetamide (HL1). For the synthesis of HL1, 1 equiv. of 2-amino- $N$-(tert-butyl)acetamide[21] $(1.75 \mathrm{~g}, 13.5 \mathrm{mmol})$ as well as $0.2 \mathrm{~g}$ of $\mathrm{Pd} / \mathrm{C}(5 \mathrm{wt} \% \mathrm{Pd})$ were added to a solution of 1 equiv. of salicylic aldehyde $(1.64 \mathrm{~g}, 13.5 \mathrm{mmol})$ in $80 \mathrm{~mL}$ of $\mathrm{MeOH}$. The resulting dark-yellowish clouded solution was subsequently stirred at $50{ }^{\circ} \mathrm{C}$ in a pressure reactor under $\mathrm{H}_{2}$ atmosphere $(5 \mathrm{~atm})$ for $24 \mathrm{~h}$. The reaction solution was subsequently filtered and the reddish filtrate evaporated in vacuo. The crude reddish oily solid was purified via column chromatography on silica gel (ethyl acetate/cyclohexane gradient from $20 \%$ to $100 \%$ ethyl acetate) to give $\mathbf{H L 1}$ as off-white solid (2.05 g, 65\%). ${ }^{1} \mathrm{H} \mathrm{NMR}\left(300 \mathrm{MHz},\left(\mathrm{CD}_{3}\right)_{2} \mathrm{SO}, 25{ }^{\circ} \mathrm{C}, \mathrm{OH} \&\right.$ amine $\mathrm{NH}$ obscured) $\delta: 7.51(\mathrm{bs}, 1 \mathrm{H}, \mathrm{NH}), 7.09-7.04(\mathrm{~m}, 2 \mathrm{H}, \mathrm{ArH}), 6.76-6.70(\mathrm{~m}, 2 \mathrm{H}, \mathrm{ArH}), 3.66\left(\mathrm{~s}, 2 \mathrm{H}, \mathrm{CH}_{2}\right), 3.02(\mathrm{~s}, 2 \mathrm{H}$, $\left.\mathrm{CH}_{2}\right), 1.25(\mathrm{~s}, 9 \mathrm{H}, t \mathrm{Bu}) \mathrm{ppm} ;{ }^{13} \mathrm{C} \mathrm{NMR}\left(75 \mathrm{MHz},\left(\mathrm{CD}_{3}\right)_{2} \mathrm{SO}, 25{ }^{\circ} \mathrm{C}\right) \delta: 169.95(\mathrm{C}=\mathrm{O}), 156.47$ (Ar-O), 129.24, 128.00, 124.95, 118.60, 115.19 (Ar), 51.39, 49.84, $49.33\left(2 \times \mathrm{CH}_{2}, \mathrm{q}-t \mathrm{Bu}\right), 28.51(t \mathrm{Bu}) \mathrm{ppm} ; \mathrm{HR}-\mathrm{MS}\left(\mathrm{ESI}^{+}\right) \mathrm{m} / \mathrm{z}$ $[\mathrm{M}+\mathrm{H}]^{+}$calcd. for $\mathrm{C}_{13} \mathrm{H}_{20} \mathrm{O}_{2} \mathrm{~N}_{2}: 273.1598$, found: 237.1594; IR (ATR, $\mathrm{cm}^{-1}$ ) ṽ: 1591 (m), 1537 (m), 1453 (m), 1391 (w), $1251(\mathrm{w}), 1224(\mathrm{~m}), 754(\mathrm{~s}), 435(\mathrm{w})$;

Synthesis of $\mathrm{N}$-(tert-butyl)-2-((3,5-di-tert-butyl-2-hydroxybenzyl)amino)acetamide (HL2). For the synthesis of HL2, 1.1 equiv. of 2-amino- $N$-(tert-butyl)acetamide[21] (1.68 g, $12.9 \mathrm{mmol})$ as well as $0.5 \mathrm{~g}$ of $\mathrm{Pd} / \mathrm{C}(5 \mathrm{wt} \% \mathrm{Pd})$ were added to a solution of 1 equiv. of 3,5-di-tert-butyl-2-hydroxybenzaldehyde $(2.75 \mathrm{~g}, 11.7 \mathrm{mmol})$ in $100 \mathrm{~mL}$ of $\mathrm{MeOH}$. The resulting yellowish clouded solution was subsequently stirred at $50{ }^{\circ} \mathrm{C}$ in a pressure reactor under $\mathrm{H}_{2}$ atmosphere ( $5 \mathrm{~atm})$ for $24 \mathrm{~h}$. The reaction solution was subsequently filtered and the pale violet filtrate evaporated in vacuo. The crude pale violet powder was purified via column chromatography on silica gel (ethyl acetate/cyclohexane gradient from $20 \%$ to $100 \%$ ethyl acetate) to give $\mathbf{H L 2}$ as white solid (2.69 g, $66 \%) .{ }^{1} \mathrm{H}$ NMR $\left(300 \mathrm{MHz},\left(\mathrm{CD}_{3}\right)_{2} \mathrm{SO}, 25^{\circ} \mathrm{C}\right) \delta: 11.44$ (bs, 1H, OH), 7.53 (bs, 1H, NH), $7.08(\mathrm{~d}, 1 \mathrm{H}, \mathrm{ArH}), 6.84(\mathrm{~d}, 1 \mathrm{H}, \mathrm{ArH}), 3.78$ (s, 2H, $\left.\mathrm{CH}_{2}\right), 3.25(\mathrm{bs}, 1 \mathrm{H}, \mathrm{NH}), 3.09\left(\mathrm{~s}, 2 \mathrm{H}, \mathrm{CH}_{2}\right), 1.35(\mathrm{~s}, 9 \mathrm{H}, t \mathrm{Bu}), 1.26(\mathrm{~s}, 9 \mathrm{H}, t \mathrm{Bu}), 1.22(\mathrm{~s}, 9 \mathrm{H}, t \mathrm{Bu}) \mathrm{ppm} ;{ }^{13} \mathrm{C}$ NMR (75 MHz, $\left.\left(\mathrm{CD}_{3}\right)_{2} \mathrm{SO}, 25^{\circ} \mathrm{C}\right) \delta: 169.24(\mathrm{C}=\mathrm{O}), 154.29(\mathrm{Ar}-\mathrm{O}), 139.35,134.46,123.21,122.49,121.67$ (Ar), 51.87, $\left.50.17\left(\mathrm{CH}_{2}\right), 50.11,34.42,33.74(\mathrm{q}-t \mathrm{Bu}), 31.55,29.54,28.55(t \mathrm{Bu}) \mathrm{ppm} ; \mathrm{HR}-\mathrm{MS}:(\mathrm{ESI})^{+}\right) \mathrm{m} / \mathrm{z}[\mathrm{M}+\mathrm{H}]^{+} \mathrm{calcd}$. for $\mathrm{C}_{21} \mathrm{H}_{36} \mathrm{O}_{2} \mathrm{~N}_{2}$ : 349.2850, found: 349.2846; IR (ATR, $\mathrm{cm}^{-1}$ ) ṽ: 1674 (s), $1542(\mathrm{~m}), 1439$ (m), 1360 (m), 1228 (m), $578(w)$ 
Synthesis of 2-((3,5-di-tert-butyl-2-hydroxybenzyl)amino)- $N$-phenylacetamide (HL3). For the synthesis of HL3, 1 equiv. of 2-amino- $N$-phenylacetamide[21] (540 mg, $3.6 \mathrm{mmol})$ as well as $120 \mathrm{mg}$ of $\mathrm{Pd} / \mathrm{C}(5 \mathrm{wt} \% \mathrm{Pd})$ were added to a solution of 1 equiv. of 3,5-di-tert-butyl-2-hydroxybenzaldehyde (842 mg, $3.6 \mathrm{mmol})$ in $50 \mathrm{~mL}$ of $\mathrm{MeOH}$. The resulting yellowish clouded solution was subsequently stirred at $50{ }^{\circ} \mathrm{C}$ in a pressure reactor under $\mathrm{H}_{2}$ atmosphere $(5$ atm) for $24 \mathrm{~h}$. The reaction solution was subsequently filtered and the brownish filtrate evaporated in vacuo. The crude beige solid was purified via column chromatography on silica gel (ethyl acetate/cyclohexane gradient from $20 \%$ to $100 \%$ ethyl acetate) to give $\mathbf{H L 3}$ as white solid (601 mg, 45\%). ${ }^{1} \mathrm{H}$ NMR $\left(300 \mathrm{MHz},\left(\mathrm{CD}_{3}\right)_{2} \mathrm{SO}, 25{ }^{\circ} \mathrm{C}\right.$, amine NH obscured) $\delta$ : 11.43 (bs, 1H, OH), 10.00 (bs, 1H, NH), 7.61 (“d”, 2H, ArH), 7.31 (“t”, 2H, ArH), 7.10 (d, 1H, ArH), 7.05 (“t”, 1H, ArH), 6.89 (d, 1H, ArH), 3.88 (s, 2H, $\left.\mathrm{CH}_{2}\right), 3.41\left(\mathrm{~s}, 2 \mathrm{H}, \mathrm{CH}_{2}\right), 1.37(\mathrm{~s}, 9 \mathrm{H}, t \mathrm{Bu}), 1.23(\mathrm{~s}$, $9 \mathrm{H}, t \mathrm{Bu}) \mathrm{ppm} ;{ }^{13} \mathrm{C} \mathrm{NMR}\left(75 \mathrm{MHz},\left(\mathrm{CD}_{3}\right)_{2} \mathrm{SO}, 25^{\circ} \mathrm{C}\right) \delta: 169.06(\mathrm{C}=\mathrm{O}), 154.28(\mathrm{Ar}-\mathrm{O}), 139.47,138.88,134.56$, $128.77(2 \mathrm{x}), 123.33,123.25,122.42,121.77,119.05(2 \mathrm{x})(\mathrm{Ar}), 51.97,50.40\left(\mathrm{CH}_{2}\right), 34.45,33.77(\mathrm{q}-\mathrm{t} \mathrm{Bu}), 31.56$, $29.55(t \mathrm{Bu})$ ppm; HR-MS: $\left(\mathrm{ESI}^{+}\right) \mathrm{m} / \mathrm{z}[\mathrm{M}+\mathrm{H}]^{+}$calcd. for $\mathrm{C}_{23} \mathrm{H}_{32} \mathrm{O}_{2} \mathrm{~N}_{2}: 369.2537$, found: 369.2534; IR (ATR, $\left.\mathrm{cm}^{-1}\right) \tilde{v}$ : 1665 (m), 1518 (s), 1481 (m), 1437 (s), 1234 (m), 1103 (w), 879 (w), 761 (s), 694 (s), 537 (w), 500 (m);

Synthesis of $N$-(tert-butyl)-2-((3,5-dichloro-2-hydroxybenzyl)amino)acetamide (HL4). For the synthesis of HL4, 1 equiv. of 2-amino- $N$-(tert-butyl)acetamide[21] (687 mg, $5.2 \mathrm{mmol})$ as well as 1 equiv. of 3,5-dichloro-2hydroxybenzaldehyde (1000 mg, $5.2 \mathrm{mmol})$ in $5 \mathrm{~mL}$ of MeOH. The initially deep yellow solution was subsequently stirred at $50{ }^{\circ} \mathrm{C}$ for $4 \mathrm{~h}$, whereupon a dark yellow precipitate started to form. The precipitate was subsequently filtered off, washed with $2 \times 5 \mathrm{~mL}$ of pentane and dried in vacuo. The resulting orange solid was subsequently dissolved in $10 \mathrm{~mL}$ of $\mathrm{MeOH}$ and 4 equiv. of $\mathrm{NaBH}_{4}(787 \mathrm{mg}, 20.8 \mathrm{mmol})$ were added portionwise, accompanied by gas evolution and decoloration of the reaction solution. Subsequently the clear reaction solution was stirred overnight at room temperature. After evaporation in vacuo, the crude oily substance was purified via column chromatography on silica gel (ethyl acetate/cyclohexane gradient from $20 \%$ to $100 \%$ ethyl acetate) to give HL4 as pale pink solid (600 mg, 38\%). ${ }^{1} \mathrm{H}$ NMR $\left(300 \mathrm{MHz},\left(\mathrm{CD}_{3}\right)_{2} \mathrm{SO}, 25{ }^{\circ} \mathrm{C}\right) \delta: 7.85$ (bs, $\left.2 \mathrm{H}, \mathrm{NH}_{2}{ }^{+}\right), 7.55$ (bs, $\left.1 \mathrm{H}, \mathrm{NH}\right)$, $7.34(\mathrm{~d}, 1 \mathrm{H}, \mathrm{ArH}), 7.10(\mathrm{~d}, 1 \mathrm{H}, \mathrm{ArH}), 3.81\left(\mathrm{~s}, 2 \mathrm{H}, \mathrm{CH}_{2}\right), 3.10\left(\mathrm{~s}, 2 \mathrm{H}, \mathrm{CH}_{2}\right), 1.26(\mathrm{~s}, 9 \mathrm{H}, t \mathrm{Bu}) \mathrm{ppm} ;{ }^{13} \mathrm{C} \mathrm{NMR}(75$ $\left.\mathrm{MHz},\left(\mathrm{CD}_{3}\right)_{2} \mathrm{SO}, 25^{\circ} \mathrm{C}\right) \delta: 169.10(\mathrm{C}=\mathrm{O}), 153.00(\mathrm{Ar}-\mathrm{O}), 127.35,127.07,127.02,121.43,120.55$ (Ar), $50.14,50.06$ $\left(\mathrm{CH}_{2}\right), 50.04(\mathrm{q}-t \mathrm{Bu}), 28.49(t \mathrm{Bu})$ ppm; HR-MS: $\left(\mathrm{ESI}^{+}\right) \mathrm{m} / \mathrm{z}[\mathrm{M}+\mathrm{H}]^{+}$calcd. for $\mathrm{C}_{13} \mathrm{H}_{18} \mathrm{O}_{2} \mathrm{~N}_{2} \mathrm{Cl}_{2}$ : 305.0818, found: 305.0815; IR (ATR, cm ${ }^{-1}$ ) ṽ: 1646 (s), 1574 (m), 1451 (s), 1297(s), 1217 (s), 1166 (m), 886 (m), 735 (s), 458 (m); 
Complex Syntheses. All dinuclear complexes are stable towards air and moisture in solid state and can be stored at ambient conditions for several weeks without decomposition. Mononuclear complexes are stable towards air but slightly sensitive towards moisture. They can be stored in a desiccator over $\mathrm{P}_{2} \mathrm{O}_{5}$ for several weeks without decomposition.

Synthesis of $\left[\left\{\mathrm{MoO}_{\mathbf{2}}(\mathbf{L 1})\right\}_{\mathbf{2}}(\boldsymbol{\mu}-\mathbf{O})\right]$ (1). For the synthesis of 1, 1 equiv. of [ $\left.\mathrm{MoO}_{2}(\mathrm{acac})_{2}\right](111 \mathrm{mg}, 0.34 \mathrm{mmol})$ was added to a solution of 1 equiv. of $\mathbf{H L 1}(80 \mathrm{mg}, 0.34 \mathrm{mmol})$ in $5 \mathrm{~mL}$ of MeCN. The orange reaction mixture was stirred for $2 \mathrm{~h}$ at $60{ }^{\circ} \mathrm{C}$ whereupon the formed dark yellow precipitate was filtered off and washed with a small amount of cold $\mathrm{MeCN}$ and twice with $5 \mathrm{~mL}$ of pentane. Evaporation of all volatiles in vacuo led to 1 as dark yellow powder $(90 \%, 113 \mathrm{mg})$. Crystals suitable for single-crystal X-ray diffraction analysis were obtained via recrystallization from a concentrated $\mathrm{MeCN}$ solution at $5{ }^{\circ} \mathrm{C} .{ }^{1} \mathrm{H} \mathrm{NMR}\left(300 \mathrm{MHz},\left(\mathrm{CD}_{3}\right)_{2} \mathrm{SO}, 25{ }^{\circ} \mathrm{C}\right.$, major isomer) $\delta: 8.40(\mathrm{~s}, 2 \mathrm{H}, \mathrm{CONH}), 7.10-7.02(\mathrm{~m}, 4 \mathrm{H}, \mathrm{ArH}), 6.67-6.62(\mathrm{~m}, 2 \mathrm{H}, \mathrm{ArH}), 6.54-6.52(\mathrm{~m}, 2 \mathrm{H}, \mathrm{ArH}), 5.50(\mathrm{~d}, 2 \mathrm{H}, \mathrm{NH})$, 4.18-4.14 (m, 2H, $\left.\mathrm{CH}_{2}\right), 4.00-3.87\left(\mathrm{~m}, 2 \mathrm{H}, \mathrm{CH}_{2}\right), 3.60-3.56\left(\mathrm{~m}, 2 \mathrm{H}, \mathrm{CH}_{2}\right), 3.14-3.08\left(\mathrm{~m}, 2 \mathrm{H}, \mathrm{CH}_{2}\right), 1.00(\mathrm{~s}, 18 \mathrm{H}$, $t \mathrm{Bu}) \mathrm{ppm} ;{ }^{1} \mathrm{H} \mathrm{NMR}\left(300 \mathrm{MHz},\left(\mathrm{CD}_{3}\right)_{2} \mathrm{SO}, 25^{\circ} \mathrm{C}\right.$, minor isomer) $\delta: 8.40(\mathrm{~s}, 2 \mathrm{H}, \mathrm{CONH}), 7.10-7.02(\mathrm{~m}, 4 \mathrm{H}, \mathrm{ArH})$, 6.67-6.62 (m, 2H, ArH), 6.54-6.52 (m, 2H, ArH), $5.18(\mathrm{~d}, 2 \mathrm{H}, \mathrm{NH}), 4.18-4.14\left(\mathrm{~m}, 2 \mathrm{H}, \mathrm{CH}_{2}\right), 4.00-3.87(\mathrm{~m}, 2 \mathrm{H}$, $\left.\mathrm{CH}_{2}\right), 3.60-3.56\left(\mathrm{~m}, 2 \mathrm{H}, \mathrm{CH}_{2}\right), 3.14-3.08\left(\mathrm{~m}, 2 \mathrm{H}, \mathrm{CH}_{2}\right), 1.02(\mathrm{~s}, 18 \mathrm{H}, t \mathrm{Bu}) \mathrm{ppm} ;{ }^{13} \mathrm{C} \mathrm{NMR}\left(75 \mathrm{MHz},\left(\mathrm{CD}_{3}\right)_{2} \mathrm{SO}_{2} 25\right.$ ${ }^{\circ} \mathrm{C}$, major isomer) $\delta$ : $173.33(\mathrm{C}=\mathrm{O}), 162.79(\mathrm{Ar}-\mathrm{O}), 129.93,129.29,123.42,119.20,118.31(\mathrm{Ar}), 51.73\left(\mathrm{CH}_{2}\right), 51.67$ (q- $t \mathrm{Bu}), 27.95(t \mathrm{Bu})$ ppm; IR (ATR, cm $\left.{ }^{-1}\right) \tilde{v}: 1624(\mathrm{~m}), 1480(\mathrm{w}), 1262(\mathrm{~m}), 930(\mathrm{~m}), 899(\mathrm{~s}), 878(\mathrm{~s}), 753$ (s, br), $726 \quad(\mathrm{~s}), \quad 617 \quad(\mathrm{~s}), \quad 496 \quad(\mathrm{~m}), \quad 478 \quad(\mathrm{~m}), \quad 388 \quad(\mathrm{w}) ; \quad$ HR-MS: $\quad\left(\mathrm{ESI}^{+}\right) \quad \mathrm{m} / \mathrm{z} \quad[\mathrm{M}+\mathrm{H}]^{+} \quad$ calcd. for $\mathrm{C}_{26} \mathrm{H}_{38} \mathrm{O}_{9} \mathrm{~N}_{4} \mathrm{Mo}_{2}$ : 743.0829, found: 743.0829; Anal. calcd. for $\mathrm{C}_{26} \mathrm{H}_{38} \mathrm{Mo}_{2} \mathrm{~N}_{4} \mathrm{O}_{9}$ : C, 42.06; H, 5.16; N, 7.55. Found: C, 42.50, H, 5.08; N, 7.37\%.

Synthesis of $\left[\mathrm{MoO}_{2}(\mathrm{L1})(\mathrm{OMe})\right]$ (2). For the synthesis of 2, 1 equiv. of $\left[\mathrm{MoO}_{2}(\mathrm{acac})_{2}\right](200 \mathrm{mg}, 0.61 \mathrm{mmol})$ was added to a solution of 1 equiv. of $\mathbf{H L 1}(142 \mathrm{mg}, 0.61 \mathrm{mmol})$ in $5 \mathrm{~mL}$ of MeOH. The yellowish reaction mixture was stirred for $4 \mathrm{~h}$ at $50{ }^{\circ} \mathrm{C}$ whereupon the formed pale yellow precipitate was filtered off and washed twice with a small amount of cold MeOH. Evaporation of all volatiles in vacuo led to 2 as pale yellow powder (86\%, $246 \mathrm{mg})$. Crystals suitable for single-crystal $\mathrm{X}$-ray diffraction analysis were obtained via recrystallization from a concentrated $\mathrm{MeOH}$ solution at $5{ }^{\circ} \mathrm{C} .{ }^{1} \mathrm{H}$ NMR $\left(300 \mathrm{MHz},\left(\mathrm{CD}_{3}\right)_{2} \mathrm{SO}, 25{ }^{\circ} \mathrm{C}\right) \delta: 8.57(\mathrm{~s}, 1 \mathrm{H}, \mathrm{CONH}), 7.12-7.05(\mathrm{~m}$, 2H, ArH), 6.69-6.64 (m, 1H, ArH), 6.57-6.55 (m, 1H, ArH), 5.92 (d, 1H, NH), 4.20-4.15 (m, 1H, $\left.\mathrm{CH}_{2}\right), 4.01(\mathrm{~s}, 3 \mathrm{H}$, OMe), 3.51-3.42 (m, 2H, $\left.\mathrm{CH}_{2}\right), 3.18-3.12\left(\mathrm{~m}, 1 \mathrm{H}, \mathrm{CH}_{2}\right), 1.00(\mathrm{~s}, 9 \mathrm{H}, t \mathrm{Bu}) ;{ }^{13} \mathrm{C} \mathrm{NMR}\left(75 \mathrm{MHz},\left(\mathrm{CD}_{3}\right)_{2} \mathrm{SO}, 25^{\circ} \mathrm{C}\right) \delta$ : 
$173.26(\mathrm{C}=\mathrm{O}), 162.33(\mathrm{Ar}-\mathrm{O}), 130.02,129.42,119.16,118.50(\mathrm{Ar}), 64.36(\mathrm{OMe}), 52.15\left(\mathrm{CH}_{2}\right), 51.86(\mathrm{q}-t \mathrm{Bu})$, $51.13\left(\mathrm{CH}_{2}\right), 27.76(t \mathrm{Bu})$; IR (ATR, cm $\left.{ }^{-1}\right)$ v: $1615(\mathrm{~s}), 1574(\mathrm{~m}), 1485(\mathrm{~m}), 1450(\mathrm{~m}), 1267(\mathrm{~m}), 1050(\mathrm{~m}), 927(\mathrm{~s})$, 878 (s), 769 (s), 620 (s), 550 (s), 500 (m), 475 (s); HR-MS: $\left(\mathrm{ESI}^{+}\right.$) m/z [M-OMe] calcd. for $\mathrm{C}_{14} \mathrm{H}_{22} \mathrm{MoN}_{2} \mathrm{O}_{5}$ : 365.0394, found: 365.0391; Anal. calcd. for $\mathrm{C}_{14} \mathrm{H}_{22} \mathrm{MoN}_{2} \mathrm{O}_{5}$ : C, 42.65; H, 5.62; N, 7.10. Found: C, 42.55, H, 5.46; $\mathrm{N}, 6.93 \%$.

Synthesis of $\left[\left\{\mathrm{MoO}_{2}(\mathbf{L 2})\right\}_{\mathbf{2}}(\boldsymbol{\mu}-\mathbf{O})\right]$ (3). For the synthesis of $\mathbf{3}, 1$ equiv. of $\left[\mathrm{MoO}_{2}(\mathrm{acac})_{2}\right](100 \mathrm{mg}, 0.31 \mathrm{mmol})$ was added to a solution of 1 equiv. of HL2 $(107 \mathrm{mg}, 0.31 \mathrm{mmol})$ in $3 \mathrm{~mL}$ of MeCN. The orange reaction mixture was stirred for $6 \mathrm{~h}$ at $60{ }^{\circ} \mathrm{C}$ whereupon the formed orange precipitate was filtered off and washed thrice with $5 \mathrm{~mL}$ of pentane. Evaporation of all volatiles in vacuo led to 3 as an orange solid (90\%, $135 \mathrm{mg})$. Crystals suitable for singlecrystal X-ray diffraction analysis were obtained via slow evaporation of a concentrated $\left(\mathrm{CD}_{3}\right)_{2} \mathrm{SO}$ solution at room temperature. ${ }^{1} \mathrm{H}$ NMR $\left(300 \mathrm{MHz},\left(\mathrm{CD}_{3}\right)_{2} \mathrm{SO}, 25{ }^{\circ} \mathrm{C}\right.$, major isomer) $\delta: 8.23(\mathrm{~s}, 2 \mathrm{H}, \mathrm{CONH}), 7.06(\mathrm{~d}, 2 \mathrm{H}, \mathrm{ArH}), 6.91$ (d, 2H, ArH), $5.55(\mathrm{~d}, 2 \mathrm{H}, \mathrm{NH}), 4.11-3.93\left(\mathrm{~m}, 4 \mathrm{H}, \mathrm{CH}_{2}\right), 3.51-3.47\left(\mathrm{~m}, 2 \mathrm{H}, \mathrm{CH}_{2}\right), 3.20-3.10\left(\mathrm{~m}, 2 \mathrm{H}, \mathrm{CH}_{2}\right), 1.28(\mathrm{~s}$, $18 \mathrm{H}, t \mathrm{Bu}), 1.23(\mathrm{~s}, 18 \mathrm{H}, t \mathrm{Bu}), 0.95(\mathrm{~s}, 18 \mathrm{H}, t \mathrm{Bu}) \mathrm{ppm} ;{ }^{1} \mathrm{H} \mathrm{NMR}\left(300 \mathrm{MHz},\left(\mathrm{CD}_{3}\right)_{2} \mathrm{SO}, 25{ }^{\circ} \mathrm{C}\right.$, minor isomer $) \delta: 8.29$ (s, 2H, CONH), 7.07 (d, 2H, ArH), 6.89 (d, 2H, ArH), 5.09 (d, 2H, NH), 4.11-3.93 (m, 4H, CH2), 3.51-3.47 (m, 2H, $\left.\mathrm{CH}_{2}\right), 3.20-3.10\left(\mathrm{~m}, 2 \mathrm{H}, \mathrm{CH}_{2}\right), 1.30(\mathrm{~s}, 18 \mathrm{H}, t \mathrm{Bu}), 1.23(\mathrm{~s}, 18 \mathrm{H}, t \mathrm{Bu}), 0.96(\mathrm{~s}, 18 \mathrm{H}, t \mathrm{Bu}) \mathrm{ppm} ;{ }^{13} \mathrm{C} \mathrm{NMR}(75 \mathrm{MHz}$, $\left(\mathrm{CD}_{3}\right)_{2} \mathrm{SO}, 25^{\circ} \mathrm{C}$, major isomer) $\delta: 173.38(\mathrm{C}=\mathrm{O}), 159.46(\mathrm{Ar}-\mathrm{O}), 139.43,136.93,124.60,122.86,122.28(\mathrm{Ar})$, 52.79, $51.51\left(\mathrm{CH}_{2}\right), 51.41,34.32,33.78(\mathrm{q}-t \mathrm{Bu}), 31.64,29.78,28.17(t \mathrm{Bu}) \mathrm{ppm}$; IR (ATR, $\left.\mathrm{cm}^{-1}\right) \tilde{v}: 1621(\mathrm{~m}), 1440$ (w), 1266 (w), 910 (m), 872 (s), 853 (s), 738 (vs, br), 554 (m), 482 (w); HR-MS: (ESI $\left.{ }^{+}\right)$m/z [M+H] calcd. for $\mathrm{C}_{42} \mathrm{H}_{70} \mathrm{Mo}_{2} \mathrm{~N}_{4} \mathrm{O}_{9}$ : 968.3343, found: 968.3335; Anal. calcd. for $\mathrm{C}_{42} \mathrm{H}_{70} \mathrm{Mo}_{2} \mathrm{~N}_{4} \mathrm{O}_{9}:$ C, 52.17; H, 7.30; N, 5.79. Found: C, 52.10, H, 6.82; N, 5.77\%.

Synthesis of $\left[\mathrm{MoO}_{2}(\mathrm{L2})(\mathrm{OMe})\right]$ (4). For the synthesis of 4, 1 equiv. of $\left[\mathrm{MoO}_{2}(\mathrm{acac})_{2}\right](100 \mathrm{mg}, 0.31 \mathrm{mmol}) \mathrm{was}$ added to a solution of 1 equiv. of HL2 $(107 \mathrm{mg}, 0.31 \mathrm{mmol})$ in $3 \mathrm{~mL}$ of MeOH. The yellowish reaction mixture was stirred for $6 \mathrm{~h}$ at $50{ }^{\circ} \mathrm{C}$ whereupon the formed yellow precipitate was filtered off and washed twice with a small amount of cold $\mathrm{MeOH}$. Evaporation of all volatiles in vacuo led to 4 as a bright yellow solid (75\%, $117 \mathrm{mg})$. Crystals suitable for single-crystal X-ray diffraction analysis were obtained via crystallization from a concentrated MeOH solution at $5{ }^{\circ} \mathrm{C} .{ }^{1} \mathrm{H} \mathrm{NMR}\left(300 \mathrm{MHz},\left(\mathrm{CD}_{3}\right)_{2} \mathrm{SO}, 25{ }^{\circ} \mathrm{C}\right) \delta: 8.48(\mathrm{~s}, 1 \mathrm{H}, \mathrm{CONH}), 7.08(\mathrm{~d}, 1 \mathrm{H}, \mathrm{ArH}), 6.93(\mathrm{~d}$, 1H, ArH), 5.92 (d, 1H, NH), 4.13-4.09 (m, 1H, $\left.\mathrm{CH}_{2}\right), 3.97$ (s, 3H, OMe), 3.46-3.38 (m, 2H, $\left.\mathrm{CH}_{2}\right), 3.21-3.16(\mathrm{~m}, 1 \mathrm{H}$, $\left.\mathrm{CH}_{2}\right), 1.29(\mathrm{~s}, 9 \mathrm{H}, t \mathrm{Bu}), 1.23(\mathrm{~s}, 9 \mathrm{H}, t \mathrm{Bu}), 0.94(\mathrm{~s}, 9 \mathrm{H}, t \mathrm{Bu}) ;{ }^{13} \mathrm{C} \mathrm{NMR}\left(75 \mathrm{MHz},\left(\mathrm{CD}_{3}\right)_{2} \mathrm{SO}, 25{ }^{\circ} \mathrm{C}\right) \delta: 173.19(\mathrm{C}=\mathrm{O})$, 
158.90 (Ar-O), 139.79, 136.92, 124.69, 122.98, 122.39 (Ar), $65.88(\mathrm{OMe}), 52.85\left(\mathrm{CH}_{2}\right), 51.69(\mathrm{q}-\mathrm{Bu}), 51.45\left(\mathrm{CH}_{2}\right)$, 34.30, $33.81(\mathrm{q}-t \mathrm{Bu}), 31.62,29.78,27.85(t \mathrm{Bu})$; IR (ATR, $\left.\mathrm{cm}^{-1}\right) \tilde{v}: 1622(\mathrm{~s}), 1471(\mathrm{w}), 1260(\mathrm{~m}), 1060(\mathrm{~m}), 1032$ (m), 906 (s), 888 (s), 846 (s), 600 (m), 567 (m), 520 (s), 484 (s); HR-MS: (ESI ${ }^{+}$) m/z [M-OMe] calcd. for $\mathrm{C}_{22} \mathrm{H}_{38} \mathrm{MoN}_{2} \mathrm{O}_{5}$ : 477.1645, found: 477.1644; Anal. calcd. for $\mathrm{C}_{22} \mathrm{H}_{38} \mathrm{MoN}_{2} \mathrm{O}_{5}: \mathrm{C}, 52.17 ; \mathrm{H}, 7.56$; N, 5.53. Found: C, $52.10, \mathrm{H}, 7.44 ; \mathrm{N}, 5.57 \%$.

Synthesis of $\left[\left\{\mathbf{M o O}_{2}(\mathbf{L 3})\right\}_{2}(\boldsymbol{\mu}-\mathbf{O})\right](\mathbf{5})$. For the synthesis of 5, 1 equiv. of $\left[\mathrm{MoO}_{2}(\mathrm{acac})_{2}\right](177 \mathrm{mg}, 0.54 \mathrm{mmol})$ was added to a solution of 1 equiv. of $\mathbf{H L 3}(200 \mathrm{mg}, 0.54 \mathrm{mmol})$ in $5 \mathrm{~mL}$ of MeCN. The orange reaction mixture was stirred for $6 \mathrm{~h}$ at $60{ }^{\circ} \mathrm{C}$ whereupon the formed orange precipitate was filtered off and washed thoroughly with $\mathrm{CH}_{2} \mathrm{Cl}_{2}$. Evaporation of all volatiles in vacuo led to 5 as an orange solid $(96 \%, 260 \mathrm{mg})$. Crystals suitable for single-crystal X-ray diffraction analysis were obtained via slow evaporation of a concentrated MeCN solution at room temperature. IR (ATR, cm $\left.{ }^{-1}\right) \tilde{v}: 1628(w), 1569(w), 1452(w), 1264(w), 1072(w), 921(\mathrm{~m}), 887(\mathrm{~s}), 853(\mathrm{~m})$, 778 (s), 752 (s), 553 (m), 491 (w); HR-MS: (ESI $\left.{ }^{+}\right)$m/z [M+H] calcd. for $\mathrm{C}_{46} \mathrm{H}_{62} \mathrm{Mo}_{2} \mathrm{~N}_{4} \mathrm{O}_{9}:$ 1008.2719, found: 1008.2713; Anal. calcd. for $\mathrm{C}_{46} \mathrm{H}_{62} \mathrm{Mo}_{2} \mathrm{~N}_{4} \mathrm{O}_{9} \cdot 0.5 \mathrm{CH}_{2} \mathrm{Cl}_{2}$ : C, 53.22; H, 6.05; N, 5.34. Found: C, 53.11, $\mathrm{H}, 6.01$; $\mathrm{N}, 5.51 \%$.

Synthesis of $\left[\mathrm{MoO}_{2}(\mathrm{L3})(\mathrm{OMe})\right](6)$. For the synthesis of 6, 1 equiv. of $\left[\mathrm{MoO}_{2}(\mathrm{acac})_{2}\right](100 \mathrm{mg}, 0.31 \mathrm{mmol})$ was added to a solution of 1 equiv. of $\mathbf{H L 3}(89 \mathrm{mg}, 0.31 \mathrm{mmol})$ in $5 \mathrm{~mL}$ of $\mathrm{MeOH}$. The yellowish reaction mixture was stirred for $6 \mathrm{~h}$ at $50{ }^{\circ} \mathrm{C}$ whereupon the formed yellow precipitate was filtered off and washed twice with a small amount of cold $\mathrm{MeOH}$. Evaporation of all volatiles in vacuo led to 6 as a bright yellow solid (82\%, $116 \mathrm{mg})$. Crystals suitable for single-crystal X-ray diffraction analysis were obtained via recrystallization from a concentrated $\mathrm{MeOH}$ solution at $5{ }^{\circ} \mathrm{C} .{ }^{1} \mathrm{H} \mathrm{NMR}\left(300 \mathrm{MHz},\left(\mathrm{CD}_{3}\right)_{2} \mathrm{SO}, 25{ }^{\circ} \mathrm{C}\right) \delta: 10.65(\mathrm{~s}, 1 \mathrm{H}, \mathrm{CONH}), 7.26-7.21(\mathrm{~m}$, 2H, ArH), 7.14-7.10 (m, 1H, ArH), 7.04-6.97 (m, 4H, ArH), 6.09 (d, 1H, NH), 4.21-4.17 (m, 1H, CH $), 4.03(\mathrm{~s}, 3 \mathrm{H}$, OMe), 3.79-3.71 (m, 1H, $\left.\mathrm{CH}_{2}\right), 3.57-3.53\left(\mathrm{~m}, 1 \mathrm{H}, \mathrm{CH}_{2}\right), 3.37-3.31\left(\mathrm{~m}, 1 \mathrm{H}, \mathrm{CH}_{2}\right), 1.23(\mathrm{~s}, 9 \mathrm{H}, t \mathrm{Bu}), 1.12(\mathrm{~s}, 9 \mathrm{H}$, $t \mathrm{Bu}) ;{ }^{13} \mathrm{C}$ NMR $\left(75 \mathrm{MHz},\left(\mathrm{CD}_{3}\right)_{2} \mathrm{SO}, 25^{\circ} \mathrm{C}\right) \delta: 173.17(\mathrm{C}=\mathrm{O}), 158.69(\mathrm{Ar}-\mathrm{O}), 140.37,137.30,135.77,128.41(2 \mathrm{x})$, 125.61, 124.86, 123.24, 122.43, $121.79(2 \mathrm{x})(\mathrm{Ar}), 66.58(\mathrm{OMe}), 52.74,51.56\left(\mathrm{CH}_{2}\right), 34.30,33.76(\mathrm{q}-\mathrm{t} \mathrm{Bu}), 31.41$, $29.69(t \mathrm{Bu})$; IR (ATR, $\left.\mathrm{cm}^{-1}\right)$ ṽ: $1627(\mathrm{~m}), 1593(\mathrm{w}), 1568(\mathrm{w}), 1062(\mathrm{w}), 923(\mathrm{~m}), 884(\mathrm{~s}), 845(\mathrm{~s}), 753(\mathrm{~m}), 598(\mathrm{~m})$, 506 (s); HR-MS: $\left(\mathrm{ESI}^{+}\right) \mathrm{m} / \mathrm{z}$ [M-OMe] ${ }^{+}$calcd. for $\mathrm{C}_{24} \mathrm{H}_{34} \mathrm{MoN}_{2} \mathrm{O}_{5}: 497.1332$, found: 497.1337, $[\mathrm{M}+\mathrm{H}]^{+}$calcd. for $\mathrm{C}_{24} \mathrm{H}_{34} \mathrm{MoN}_{2} \mathrm{O}_{5}:$ 529.1600, found: 529.1597; Anal. calcd. for $\mathrm{C}_{24} \mathrm{H}_{34} \mathrm{MoN}_{2} \mathrm{O}_{5}$ : C, 54.75; H, 6.51; N, 5.32. Found: C, $54.63, \mathrm{H}, 6.03 ; \mathrm{N}, 5.43 \%$. 
Synthesis of $\left[\left\{\mathbf{M o O}_{2}(\mathbf{L 4})\right\}_{\mathbf{2}}(\boldsymbol{\mu}-\mathbf{O})\right]$ (7). For the synthesis of 7, 1 equiv. of $\left[\mathrm{MoO}_{2}(\mathrm{acac})_{2}\right](107 \mathrm{mg}, 0.33 \mathrm{mmol})$ was added to a solution of 1 equiv. of HL4 $(100 \mathrm{mg}, 0.33 \mathrm{mmol})$ in $5 \mathrm{~mL}$ of MeCN. The yellow reaction mixture was stirred for $4 \mathrm{~h}$ at $60{ }^{\circ} \mathrm{C}$ whereupon the formed bright yellow precipitate was filtered off and dried in vacuo to give 7 as a pale yellow solid $(82 \%, 119 \mathrm{mg}) .{ }^{1} \mathrm{H}$ NMR $\left(300 \mathrm{MHz},\left(\mathrm{CD}_{3}\right)_{2} \mathrm{SO}, 25{ }^{\circ} \mathrm{C}\right.$, major isomer) $\delta: 8.63(\mathrm{~s}, 2 \mathrm{H}, \mathrm{CONH})$, $7.31(\mathrm{~d}, 2 \mathrm{H}, \mathrm{ArH}), 7.18$ (d, 2H, ArH), 5.76 (d, 2H, NH), 4.10-4.01 (m, 2H, $\left.\mathrm{CH}_{2}\right), 3.92-3.77$ (m, 2H, $\left.\mathrm{CH}_{2}\right), 3.71-3.67$ $\left(\mathrm{m}, 2 \mathrm{H}, \mathrm{CH}_{2}\right), 3.19-3.13\left(\mathrm{~m}, 2 \mathrm{H}, \mathrm{CH}_{2}\right), 1.07(\mathrm{~s}, 18 \mathrm{H}, t \mathrm{Bu}) \mathrm{ppm} ;{ }^{1} \mathrm{H}$ NMR $\left(300 \mathrm{MHz},\left(\mathrm{CD}_{3}\right)_{2} \mathrm{SO}, 25{ }^{\circ} \mathrm{C}\right.$, minor isomer) $\delta: 8.56(\mathrm{~s}, 2 \mathrm{H}, \mathrm{CONH}), 7.30(\mathrm{~d}, 2 \mathrm{H}, \mathrm{ArH}), 7.16(\mathrm{~d}, 2 \mathrm{H}, \mathrm{ArH}), 5.53(\mathrm{~d}, 2 \mathrm{H}, \mathrm{NH})$, ), 4.10-4.01 (m, 2H, $\left.\mathrm{CH}_{2}\right)$, 3.92-3.77 (m, 2H, CH$), 3.63-3.59\left(\mathrm{~m}, 2 \mathrm{H}, \mathrm{CH}_{2}\right), 3.19-3.13\left(\mathrm{~m}, 2 \mathrm{H}, \mathrm{CH}_{2}\right), 1.05(\mathrm{~s}, 18 \mathrm{H}, t \mathrm{Bu}) \mathrm{ppm} ;{ }^{13} \mathrm{C} \mathrm{NMR}(75$ $\mathrm{MHz},\left(\mathrm{CD}_{3}\right)_{2} \mathrm{SO}, 25^{\circ} \mathrm{C}$, major isomer) $\delta: 173.62(\mathrm{C}=\mathrm{O}), 157.81$ (Ar-O), 128.49, 128.36, 124.57, 120.89 (Ar), 52.79 , $52.03(\mathrm{q}-t \mathrm{Bu}), 51.00\left(2 \times \mathrm{CH}_{2}\right), 27.81(t \mathrm{Bu}) \mathrm{ppm}$; IR (ATR, $\left.\mathrm{cm}^{-1}\right) \tilde{v}: 1622(\mathrm{~s}), 1458(\mathrm{~m}), 922(\mathrm{~s}), 899(\mathrm{~s}), 785(\mathrm{~s})$, 750 (vs, br), 563 (w), 478 (w); HR-MS: (ESI ${ }^{+}$) m/z [M+H] $]^{+}$calcd. for $\mathrm{C}_{26} \mathrm{H}_{34} \mathrm{Cl}_{4} \mathrm{Mo}_{2} \mathrm{~N}_{4} \mathrm{O}_{9}: 882.9238$, found: 882.9232; Anal. calcd. for $\mathrm{C}_{26} \mathrm{H}_{34} \mathrm{Cl}_{4} \mathrm{Mo}_{2} \mathrm{~N}_{4} \mathrm{O}_{9}$ : C, 35.48; H, 3.89; N, 6.36. Found: C, 35.29, H, 3.90; N, $6.15 \%$

\section{Results and discussion}

\subsection{Ligand synthesis}

Ligands HL1-HL3 were prepared according to Scheme 1. Thus, the respective hydroxybenzaldehydes were reacted with functionalized amidoamines under $\mathrm{H}_{2}$ atmosphere (5 atm) in a pressure reactor over $\mathrm{Pd} / \mathrm{C}$. The products were obtained in fair yields after purification via column chromatography.<smiles>[R]NC(=O)CN</smiles><smiles>[R]NC(=O)CNCc1cc([R1])cc([R1])c1O</smiles>

$H L 1 R^{1}=H, R^{2}=t B u(65 \%)$

$\mathrm{HL} 2 \mathrm{R}^{1}=\mathrm{R}^{2}=t \mathrm{Bu}(66 \%)$

HL3 $R^{1}=t B u, R^{2}=P h(45 \%)$

Scheme 1. Synthesis of the aminophenolate amide ligands HL1-HL3. 
Ligand HL4 was prepared in a two-step procedure according to Scheme 2. In the first step, 2hydroxy-3,5 dichlorobenzaldehyde was condensed with the respective amine. The formed Schiff base was subsequently treated with an excess of sodium borohydride. Column chromatography gave the desired product in fair yield. A synthesis procedure using $\mathrm{H}_{2}$ and $\mathrm{Pd} / \mathrm{C}$ did not yield an isolable amount of HL4.

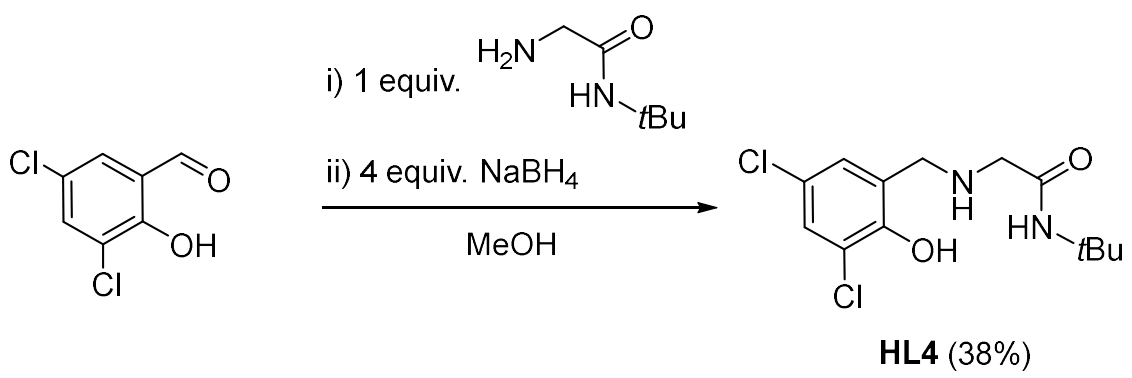

Scheme 2. Synthesis of the aminophenolate amide ligand HL4.

The employed functionalized primary amines were prepared according to published procedures.[21] Ligands HL1-HL4 were characterized via ${ }^{1} \mathrm{H},{ }^{13} \mathrm{C}$ NMR, FT-IR and HR-MS $\left(\mathrm{ESI}^{+}\right)$spectroscopy. Noteworthy is the difference in the shift of the amide NH proton, depending on the substituent (7.53 ppm in HL2 and $10.00 \mathrm{ppm}$ in HL3, tert-butyl vs. phenyl amide, $\left.\left(\mathrm{CD}_{3}\right)_{2} \mathrm{SO}\right)$. Another interesting feature arises from a comparison of the ${ }^{1} \mathrm{H}$ NMR spectra of HL1-HL4 in $\left(\mathrm{CD}_{3}\right)_{2} \mathrm{SO}$. Whereas the $\mathrm{OH}$ and $\mathrm{NH}$ protons are not observable in ligands HL1 and HL2, likely due to fast exchange, they are clearly assignable for ligand HL3. For the dichlorosubstituted ligand HL4 on the other hand ${ }^{1} \mathrm{H}$ NMR spectroscopy in $\left(\mathrm{CD}_{3}\right)_{2} \mathrm{SO}$ displays resonances which are assignable to an $\mathrm{O}^{-} \mathrm{NH}_{2}{ }^{+}$form, suggesting a zwitterionic behavior in solution (Fig. S1). Ligands HL1-HL4 feature electron withdrawing and donating substituents at the phenolate and amide moieties, respectively, allowing for an assessment of electronic influences on the properties of the resulting molybdenum complexes.

\subsection{Complex synthesis}


The synthesis of the dinuclear complexes $\mathbf{1}, \mathbf{3}, \mathbf{5}$ and $\mathbf{7}$ of the type $\left[\left\{\mathrm{MoO}_{2} \mathbf{L}\right\}_{2}(\mu-\mathrm{O})\right]$ was accomplished via the reaction of one equiv. of HL1-HL4, respectively, with one equiv. of $\left[\mathrm{MoO}_{2}(\mathrm{acac})_{2}\right]$ in $\mathrm{MeCN}$ under ambient conditions in excellent yields (Scheme 3).

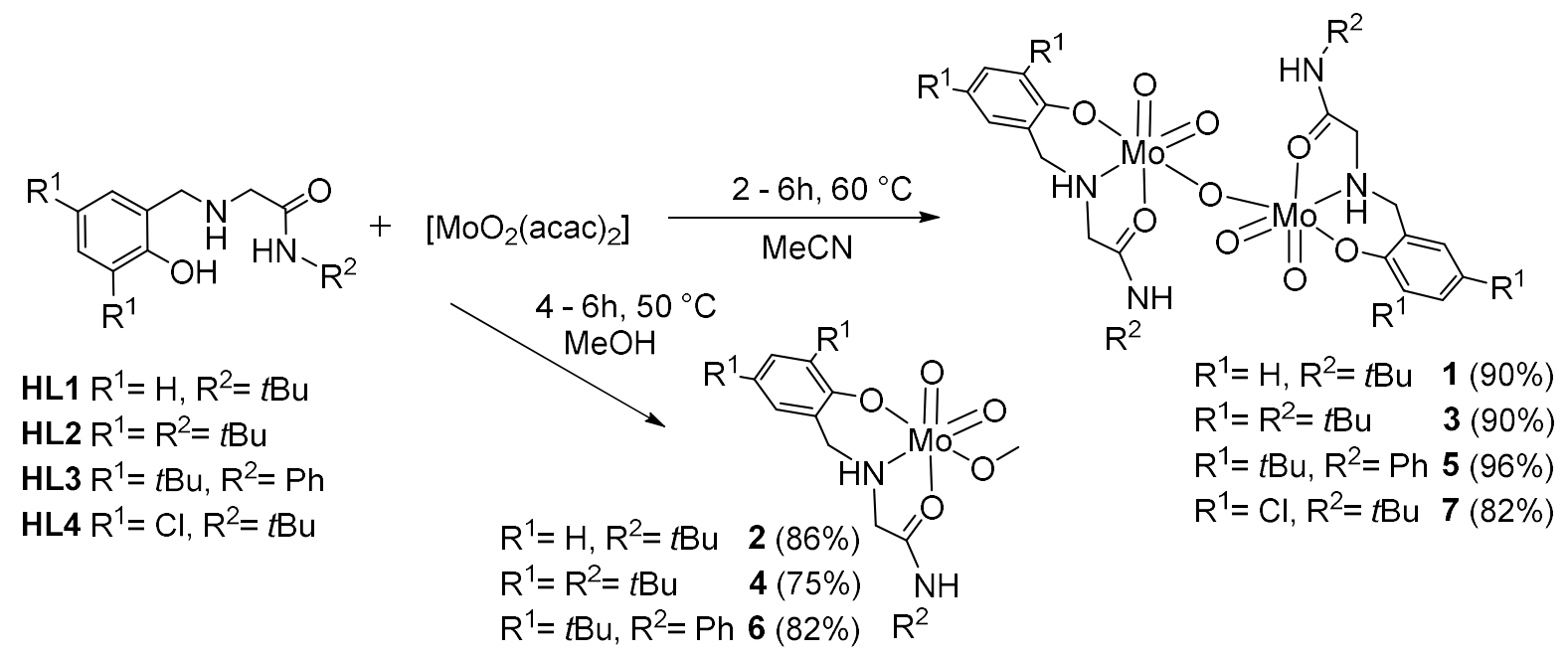

Scheme 3. Synthesis of complexes 1-7.

The bridging oxygen atom likely originates from the deprotonation of advantageous water molecules, possibly via a hydroxido intermediate, as previously disclosed for related complexes.[18] This assumption is corroborated by the formation of only traces of complex $\mathbf{3}$, accompanied by insoluble solids, if the synthesis is carried out under inert conditions in dry $\mathrm{MeCN}$. Interestingly, if the protic solvent $\mathrm{MeOH}$ is employed in the synthesis, complexes 2, 4 and $\mathbf{6}$ of the general formula $\left[\mathrm{MoO}_{2} \mathbf{L}(\mathrm{OMe})\right]$ were obtained in good to excellent yield both under ambient and inert conditions (Scheme 3). The formation of this mononuclear complexes marks an interesting contrast to previous observations, where the use of standard grade methanol also led to dinuclear compounds.[18] We attribute the formation of the mononuclear complexes to the similar acidity of the protons in $\mathrm{H}_{2} \mathrm{O}$ and $\mathrm{MeOH}$ and the vast excess of the latter in $\mathrm{MeOH}$ solution.[26] If less acidic alcoholic solvents are employed (e.g. $i \mathrm{PrOH}, t \mathrm{BuOH})$, no formation of 
the respective alcoholate complexes could be observed. The higher steric demand of an isopropyl or tert-butyl group could also inhibit the formation of such complexes.

Complexes 1-7 feature one ligand moiety per molybdenum center, coordinated in a facial ONO motif via the anionic phenolate, the amine and the carbonyl oxygen of the amide, as observed in the solid state structures of $\mathbf{1}-\mathbf{3}, \mathbf{5}$ and $\mathbf{6}$. The ${ }^{1} \mathrm{H}$ NMR spectra of the methanolate complexes $\mathbf{2}, \mathbf{4}$ and 6 feature the expected one set of shifted ligand resonances, including a broadened doublet for the proton at the coordinated amine. Interestingly, in the spectra of the dinuclear $\mu$-oxido complexes two sets of ligand resonances with a solvent dependent ratio (e.g. $3: 2$ in $\left(\mathrm{CD}_{3}\right)_{2} \mathrm{CO}$ and 5:2 in $\left(\mathrm{CD}_{3}\right)_{2} \mathrm{SO}$ for complex $\mathbf{3}$, Fig. S4) were observed. Dissolving single crystals of $\mathbf{3}$ and immediate ${ }^{1} \mathrm{H}$ NMR measurement resulted in analogous spectra, hinting towards a dynamic isomerization in solution. Considering free rotation around the $\mu$-oxido Mo-O bonds, X-ray data suggests two homotopic half units (vide infra), which would result in only one signal set observable by NMR spectroscopy. If the two halves would be non-homotopic, two signal sets in a ratio of 1:1 should result. To gain further insight, we performed VT-NMR measurements in $\left(\mathrm{CD}_{3}\right)_{2} \mathrm{SO}\left(20{ }^{\circ} \mathrm{C}\right.$ to $\left.60{ }^{\circ} \mathrm{C}\right)$ as well as in $\mathrm{CD}_{3} \mathrm{CN}\left(-30{ }^{\circ} \mathrm{C}\right.$ to $60{ }^{\circ} \mathrm{C}$, Fig. S2) which unfortunately did not show any meaningful change in the ratio of the signal sets and thus do not allow for clear assertion of the dynamics in solution. However, it is possible that the observed resonances arise from a labile coordination of the amide moieties, resulting in an equilibrium between five- and a sixfold coordinated metal centers.

We were interested if the different complexes formed in $\mathrm{MeCN}$ and $\mathrm{MeOH}$ are able to interconvert. For this reason, the following observation is of importance. If the dinuclear complexes are dissolved in $\mathrm{MeOH}$, the color of the solution brightened within minutes and removal of the solvent after $24 \mathrm{~h}$ of standing at room temperature yielded the respective 
methanolate complex in quantitative yield. A similar effect was observed upon dissolving the methanolate complexes in benchtop MeCN (Scheme 4).

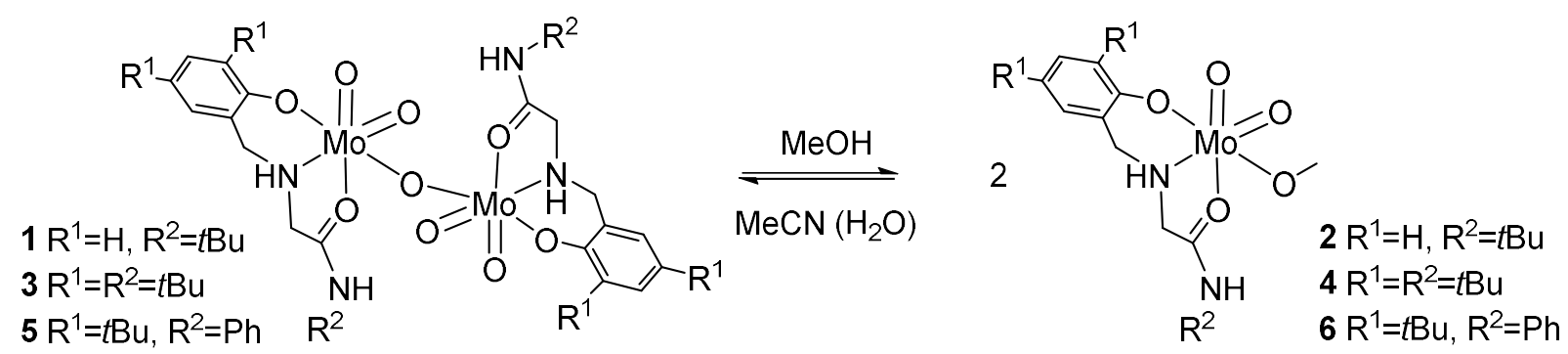

Scheme 4. Interconversion of mononuclear and dinuclear molybdenum complexes.

To obtain insight into the behavior of the complexes under catalytic conditions (vide infra), a mixture of complex 3 and ten equiv. of TBHP was dissolved in benchtop $\mathrm{CD}_{3} \mathrm{CN}$ (the solubility in $\mathrm{CDCl}_{3}$ was not sufficient to obtain meaningful spectra) and stirred for $24 \mathrm{~h}$ at $50{ }^{\circ} \mathrm{C}$. A ${ }^{1} \mathrm{H}$ NMR spectrum of the reaction solution recorded after $24 \mathrm{~h}$ showed no observable difference to the spectrum of the initial complex (Fig. S3), revealing a surprisingly high stability under these conditions. The NMR measurement repeated after leaving the sample for 30 days under ambient conditions and also after subsequent addition of $\sim 500$ equiv. of $\mathrm{H}_{2} \mathrm{O}$ still showed no substantial decomposition, again in contrast to other published precatalysts. $[9,12,17]$

\subsection{Molecular Structures}

Molecular structures of dinuclear complexes $\mathbf{1 , 3}$ and $\mathbf{5}$, as well as mononuclear complexes $\mathbf{2}$ and $\mathbf{6}$ were determined by single-crystal X-ray diffraction analysis. For complex 3, racemic twinning was observed, and for $\mathbf{6}$ two independent molecules (labelled $\mathbf{A}$ and $\mathbf{B}$, only molecule $\mathbf{A}$ discussed here, for $\mathbf{B}$ please refer to the SI) with distinct structural differences were found in the solid state. Molecular views of 1, 3 and $\mathbf{5}$ are given in Fig. 1, and of $\mathbf{2}$ and $\mathbf{6}$ in Fig. 2. Selected crystallographic data for complexes 1-3 and 5-6 are provided in Table 1, selected bond lengths 
and angles are given in Table 2, full crystallographic details such as intra- and intermolecular hydrogen bonding, structure refinement and experimental details are provided within the SI.

Table 1. Selected crystallographic data and structure refinement for complexes 1-3, 5 and $\mathbf{6}$.

\begin{tabular}{|c|c|c|c|c|c|}
\hline & 1 & 2 & $3^{[\mathrm{a}]}$ & 5 & $6^{[a]}$ \\
\hline Empirical formula & $\mathrm{C}_{26} \mathrm{H}_{38} \mathrm{Mo}_{2} \mathrm{~N}_{4} \mathrm{O}_{9}$ & $\mathrm{C}_{14} \mathrm{H}_{22} \mathrm{MoN}_{2} \mathrm{O}_{5}$ & $\begin{array}{l}\mathrm{C}_{42} \mathrm{H}_{70} \mathrm{Mo}_{2} \mathrm{~N}_{4} \mathrm{O}_{9} \cdot \\
3 \mathrm{C}_{2} \mathrm{H}_{6} \mathrm{OS}\end{array}$ & $\begin{array}{l}\mathrm{C}_{46} \mathrm{H}_{62} \mathrm{Mo}_{2} \mathrm{~N}_{4} \mathrm{O}_{9} \\
3 \mathrm{C}_{2} \mathrm{H}_{3} \mathrm{~N}\end{array}$ & $\begin{array}{l}\mathrm{C}_{24} \mathrm{H}_{34} \mathrm{MoN}_{2} \mathrm{O}_{5} \\
\mathrm{CH}_{3} \mathrm{OH}\end{array}$ \\
\hline Formula weight & 742.48 & 394.27 & 1201.28 & 1130.03 & 558.51 \\
\hline Crystal description & needle, yellow & needle, colorless & block, yellow & block, yellow & block, yellow \\
\hline $\begin{array}{l}\text { Crystal system, space } \\
\text { group }\end{array}$ & $\begin{array}{l}\text { monoclinic, } \\
\text { C } 2 / \mathrm{c}\end{array}$ & $\begin{array}{l}\text { monoclinic, } \\
\mathrm{P} 2_{1} / \mathrm{c}\end{array}$ & $\begin{array}{l}\text { orthorhombic, } \\
\text { P } 2_{1} 2_{1} 2_{1}\end{array}$ & triclinic, $\mathrm{P}-1$ & triclinic, $\mathrm{P}-1$ \\
\hline $\mathrm{a}[\AA]$ & $35.8689(16)$ & $12.734(2)$ & $11.7746(6)$ & $10.3527(7)$ & $9.5849(9)$ \\
\hline $\mathrm{b}[\AA]$ & $7.3863(3)$ & $6.8362(12)$ & $29.8324(15)$ & $15.5833(12)$ & $16.7996(17)$ \\
\hline$C[\AA]$ & $27.2284(12)$ & $18.769(4)$ & $33.3041(17)$ & $18.3910(13)$ & $18.4598(18)$ \\
\hline $\mathrm{a}\left[{ }^{\circ}\right]$ & & & & $73.523(3)$ & $113.094(3)$ \\
\hline$\beta\left[^{\circ}\right]$ & $115.7437(13)$ & $91.201(5)$ & & $77.913(4)$ & $95.732(4)$ \\
\hline$\gamma\left[^{\circ}\right]$ & & & & $75.891(3)$ & $98.174(3)$ \\
\hline Volume $\left[\AA^{3}\right]$ & $6497.8(5)$ & $1633.5(5)$ & $11698.5(10)$ & $2727.8(3)$ & $2666.6(5)$ \\
\hline Z & 8 & 4 & 8 & 2 & 4 \\
\hline $\begin{array}{l}\text { Reflections collected / } \\
\text { unique }\end{array}$ & $51941 / 9491$ & 18825 / 3211 & $63089 / 22972$ & 64481 / 24013 & 152692 / 23463 \\
\hline $\mathrm{R}($ int $), \mathrm{R}$ (sigma) & $0.0390,0.0339$ & $0.0493,0.0518$ & $0.0452,0.0769$ & $0.0537,0.0604$ & $0.0438,0.0266$ \\
\hline Completeness $\left(\Theta_{\max }\right)$ & $100.0 \%\left(30.0^{\circ}\right)$ & $99.9 \%\left(26.0^{\circ}\right)$ & $99.8 \%\left(26.0^{\circ}\right)$ & $100.0 \%\left(35.0^{\circ}\right)$ & $99.9 \%\left(35.0^{\circ}\right)$ \\
\hline Data/parameters/restraints & $9491 / 404 / 4$ & $3211 / 220 / 2$ & $22972 / 1349 / 74$ & 24013 / 685 / 4 & 23463 / 677 / 6 \\
\hline $\begin{array}{l}\text { Signif. unique refl. }[\mathrm{I}> \\
2 \sigma(\mathrm{I})]\end{array}$ & 8077 & 2621 & 18476 & 19319 & 20033 \\
\hline Goodness-of-fit on $\mathrm{F}^{2}$ & 1.040 & 1.144 & 1.114 & 1.037 & 1.047 \\
\hline Final $R$ indices $[I>2 \sigma(I)]$ & $\begin{array}{l}\mathrm{R} 1=0.0264, \\
\mathrm{wR} 2=0.0580\end{array}$ & $\begin{array}{l}\mathrm{R} 1=0.0402, \\
\mathrm{wR} 2=0.0728\end{array}$ & $\begin{array}{l}\mathrm{R} 1=0.0546 \\
\mathrm{wR} 2=0.1420\end{array}$ & $\begin{array}{l}R 1=0.0344 \\
w R 2=0.0833\end{array}$ & $\begin{array}{l}R 1=0.0239 \\
W R 2=0.0577\end{array}$ \\
\hline $\mathrm{R}$ indices (all data) & $\begin{array}{l}\mathrm{R} 1=0.0353 \\
\mathrm{wR} 2=0.0603\end{array}$ & $\begin{array}{l}\mathrm{R} 1=0.0574 \\
\mathrm{wR} 2=0.0824\end{array}$ & $\begin{array}{l}\mathrm{R} 1=0.0678 \\
\mathrm{wR} 2=0.1480\end{array}$ & $\begin{array}{l}R 1=0.0477 \\
W R 2=0.0893\end{array}$ & $\begin{array}{l}R 1=0.0332 \\
W R 2=0.0631\end{array}$ \\
\hline $\begin{array}{l}\text { Largest difference peak } \\
\text { and hole }\left[\mathrm{e} / \AA^{3}\right]\end{array}$ & 0.746 and -0.516 & 0.845 and -0.828 & 1.276 and -1.098 & $\begin{array}{l}0.890 \text { and - } \\
1.415\end{array}$ & 0.836 and -0.667 \\
\hline CCDC - Number & 1532978 & 1532979 & 1532980 & 1532981 & 1532982 \\
\hline
\end{tabular}

\footnotetext{
${ }^{[a]}$ Parameters corresponding to the second observed modification are provided within the SI.
} 

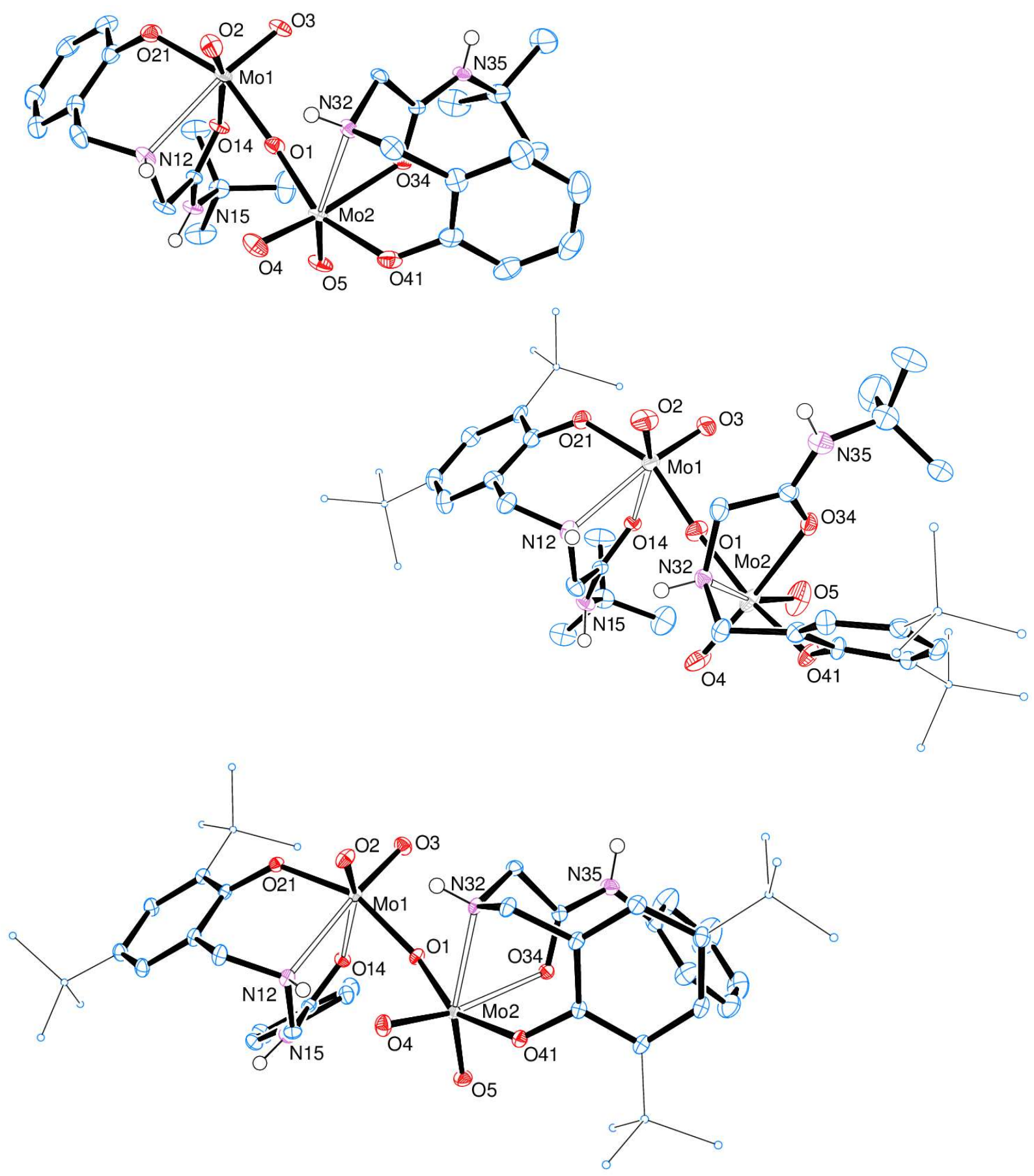

Fig. 1. Molecular views (50\% probability level) of $\mathbf{1}$ (top), $\mathbf{3}$ (middle) and $\mathbf{5}$ (bottom); H atoms (except nitrogen bound protons) as well as solvent molecules are omitted for clarity reasons. Open bonds depict unusual long Mo-L contacts. The tert-butyl substituents on the phenyl rings in $\mathbf{3}$ and $\mathbf{5}$ are drawn with small spherical atoms and line bonds for clarity reasons. 

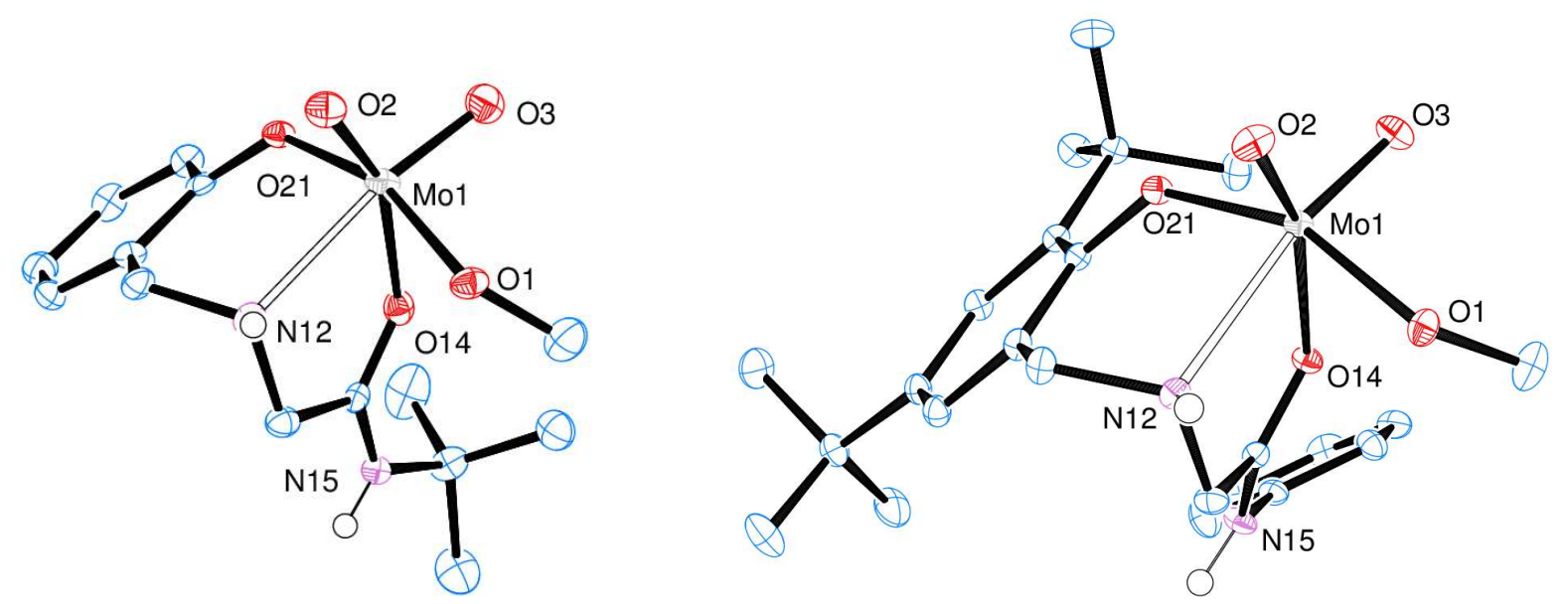

Fig. 2. Molecular views (50\% probability level) of 2 (left), and 6 (right); H atoms (except nitrogen bound protons) as well as solvent molecules are omitted for clarity reasons. Open bonds depict unusual long Mo-L contacts.

In the $\mu$-oxido bridged dinuclear complexes $\mathbf{1 , 3}$ and $\mathbf{5}$ each molybdenum center is coordinated in a distorted octahedral fashion by two oxido ligands, one facially ONO-coordinating tridentate ligand as well as the bridging oxygen atom. General features of these complexes are the rather long distances between the molybdenum atoms and the bonding amino and carbonyl groups, respectively (Table 2). Despite the similarity of these complexes, clear differences can be observed with respect to the Mo1-O1-Mo2 angle, i.e. the linearity of the $\mu$-oxido bridge, as well as the dihedral angles between the oxido groups on Mo1 and Mo2 (e.g. O2-Mo1-Mo2-O4). Whereas the Mo1-O1-Mo2 angles in 1 and 3 exhibit smaller deviations from $180^{\circ}\left(166.94(7)^{\circ}\right.$ in 1 and 175.2(4)/170.1(3) ${ }^{\circ}$ in 3), the Mo1-O1-Mo2 angle observed for complex 5 is $146.96(5)^{\circ}$. Both, linear (Mo-O-Mo angle close to $180^{\circ}$ ) and bent structures $\left(\mathrm{Mo}-\mathrm{O}-\mathrm{Mo}\right.$ angle $<180^{\circ}$ ) have been observed in previously reported dinuclear dioxidomolybdenum(VI) complexes.[18] Also, whereas the dihedral O-Mo-Mo-O angles in complexes $\mathbf{1}$ and $\mathbf{5}$ are comparable $\left(300^{\circ}\right.$ and $\left.302^{\circ}\right)$, the complex half units in $\mathbf{3}$ are rotated around the $\mu$-oxido bridge $\left(243^{\circ}\right)$, further corroborating the geometric flexibility of the complexes (Table 2). 
In the mononuclear complexes $\mathbf{2}$ and $\mathbf{6}$, the molybdenum center is coordinated in a distorted octahedral fashion that closely resembles the coordination environment of the related dinuclear compounds $\mathbf{1}$ and $\mathbf{5}$. Instead of the $\mu$-oxido bridge, the sixth coordination site in complexes $\mathbf{2}$ and 6 is occupied by a monoanionic methoxide ligand. Most bond lengths are similar to the respective dinuclear complexes with the exception of the Mo1-O1 bond to the methoxido ligand which is longer than to the $\mu$-oxido bridging atom. In general, the observed molybdenum oxido bond lengths are within expected ranges for all reported complexes (Table 2).[27] For all structurally characterized complexes, inter- and intra-molecular hydrogen bonding is observed (Tables S4, S7, S10, S13, S16 and S19). For dinuclear complexes 1, 3 and 5 intra-molecular hydrogen bonding is especially pronounced in $\mathbf{5}$, and less pronounced in $\mathbf{1}$ and $\mathbf{3}$. In these complexes, hydrogen bonds are spanning the two halves of the molecule across the $\mu$-oxido bridging atom, between the amine $\mathrm{N}-H$ atom and a terminal oxido ligand (Fig. S12). In addition, all three complexes 1, $\mathbf{3}$ and $\mathbf{5}$ show inter-molecular hydrogen bonding to neighboring complexes in the solid state. For mononuclear complexes $\mathbf{2}$ and $\mathbf{6}$, hydrogen bonds between the amide N-H atom and the methoxide oxygen atom are observed, validating the ability of ligands HL1-4 to provide hydrogen bonding. 
Table 2. Selected bond lengths $[\AA]$ and angles $\left[{ }^{\circ}\right]$ for complexes $\mathbf{1}-\mathbf{3}, \mathbf{5}$ and $\mathbf{6}$.

\begin{tabular}{lccccc}
\hline & $\mathbf{1}$ & $\mathbf{2}$ & $\mathbf{3}^{[\mathrm{a}]}$ & $\mathbf{5}$ & $\mathbf{6}^{[\mathrm{a}]}$ \\
\hline Mo1-O1 & $1.8994(12)$ & $1.923(2)$ & $1.885(5)$ & $1.9049(9)$ & $1.9237(8)$ \\
Mo1-O2 & $1.7149(12)$ & $1.710(3)$ & $1.707(6)$ & $1.7245(9)$ & $1.7172(8)$ \\
Mo1-O3 & $1.7202(11)$ & $1.728(3)$ & $1.715(5)$ & $1.7122(10)$ & $1.7221(8)$ \\
Mo1-O21 & $1.9555(12)$ & $2.002(2)$ & $1.979(5)$ & $1.9791(9)$ & $1.9520(7)$ \\
Mo1-O14 & $2.2231(11)$ & $2.227(3)$ & $2.307(5)$ & $2.2613(9)$ & $2.2257(7)$ \\
Mo1-N12 & $2.3952(13)$ & $2.361(3)$ & $2.355(5)$ & $2.3623(11)$ & $2.3617(9)$ \\
Mo2-O1 & $1.8795(12)$ & - & $1.919(5)$ & $1.9141(9)$ & - \\
Mo2-O4 & $1.7131(12)$ & - & $1.723(6)$ & $1.7178(10)$ & $1.7137(8)$ \\
Mo2-O5 & $1.7180(12)$ & - & $1.721(5)$ & $1.7129(10)$ & $1.7245(8)$ \\
Mo2-O6 & - & - & - & - & $1.9333(8)$ \\
Mo2-O41 & $1.9797(13)$ & - & $1.987(5)$ & $1.9807(9)$ & $1.9570(7)$ \\
Mo2-O34 & $2.2222(11)$ & - & $2.245(5)$ & $2.2929(9)$ & $2.2833(7)$ \\
Mo2-N32 & $2.3915(13)$ & - & $2.390(4)$ & $2.3650(11)$ & $2.3378(8)$ \\
Mo1-O1-Mo2 & $166.94(7)$ & - & $175.2(4)$ & $146.96(5)$ & - \\
O2-Mo1-O3 & $105.20(6)$ & $107.11(13)$ & $105.6(3)$ & $105.97(5)$ & $107.11(4)$ \\
O4-Mo2-O5 & $105.19(6)$ & - & $105.7(3)$ & $106.79(5)$ & $107.89(4)$ \\
O2-Mo1-Mo2-O4 & $300.94(6)$ & - & $243.03(27)$ & $302.10(5)$ & - \\
O2-Mo1-Mo2-O5 & $194.55(7)$ & - & $135.46(27)$ & $196.51(5)$ & - \\
\hline
\end{tabular}

${ }^{[a]}$ Only data for one molecule shown, bond lengths and angles for other molecule shown in SI.

\subsection{Olefin Epoxidation}

Complexes 1-7 were screened in the catalytic epoxidation of various internal and terminal olefins using two equiv. of tert-butylhydroperoxide (TBHP, 5.5 $\mathrm{M}$ in decane) or hydrogen peroxide $\left(\mathrm{H}_{2} \mathrm{O}_{2}, 30 \%\right.$ in water) as oxygen source (Fig. 3). All catalyst loadings as well as TONs are calculated per complex molecule (vide infra). Blank reactions without added catalysts were performed for all substrates and showed conversions below $10 \%$ (uncatalyzed reactions).

In an initial round, the five substrates S1-S5 were tested under previously optimized reaction conditions $\left(50{ }^{\circ} \mathrm{C}, 2\right.$ equiv. oxidant, $\left.\mathrm{CHCl}_{3}\right)$. 


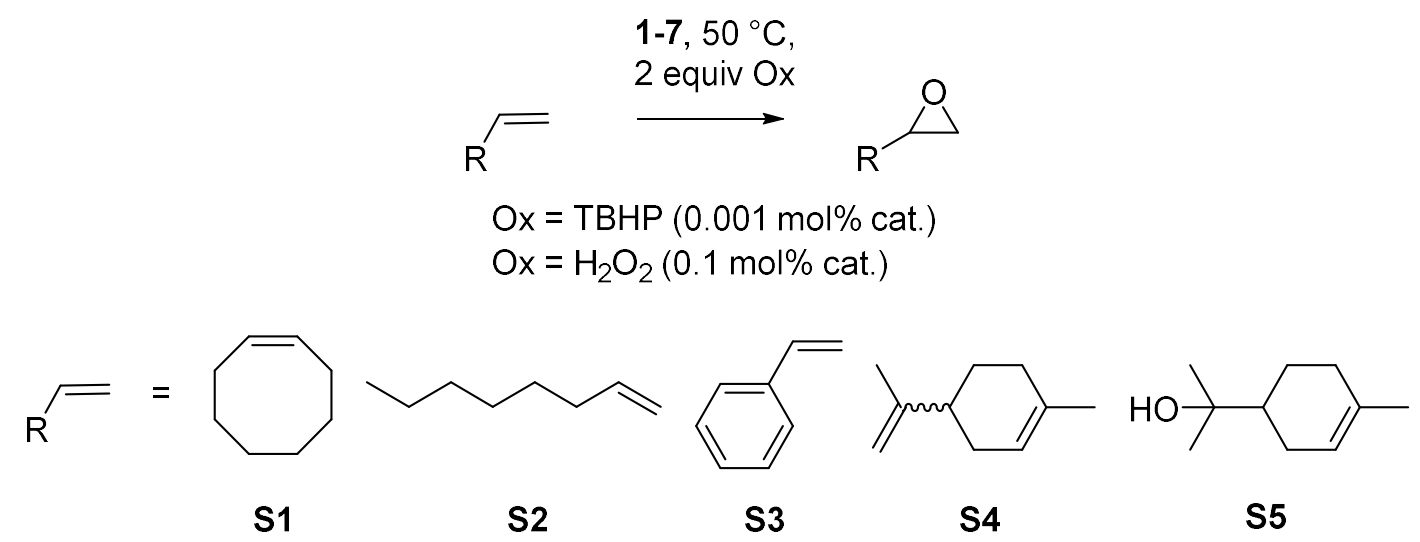

Fig. 3. Epoxidations of olefinic substrates S1-S5 by complexes 1-7.

With TBHP as oxidant, all complexes reached average to high substrate conversions, even at very low precatalyst loadings of $0.001 \mathrm{~mol} \%$, except for substrate $\mathbf{S 2}$ (Table 3). For 1-octene S2, only complex 3 and 7 gave low conversions under these conditions. For aqueous oxidant $\mathrm{H}_{2} \mathrm{O}_{2}$, only complexes $1,3,4$ and 5 gave noticeable conversions at the higher precatalyst loading of 0.1 mol\% (Table 4). A comparison of turnover numbers (TON) for all seven complexes with both oxidants is given in Table 5 .

Table 3. Overview of conversion of substrate (selectivity to epoxide) with TBHP as oxidant.

\begin{tabular}{cccccccc}
\hline & $\mathbf{1}$ & $\mathbf{2}$ & $\mathbf{3}$ & $\mathbf{4}$ & $\mathbf{5}$ & $\mathbf{6}$ & $\mathbf{7}$ \\
\hline S1 & $59(92)$ & $41(89)$ & $>95(>95)^{[\mathrm{a}]}$ & $68(>95)$ & $75(91)$ & $79(>95)$ & $>95(>95)^{[\mathrm{b}]}$ \\
S2 & no conv. & no conv. & $33(70)$ & no conv. & no conv. & no conv. & $32(92)$ \\
S3 & unsel. & $61(8)$ & $69(22)$ & unsel. & unsel. & $83(34)$ & $27(30)$ \\
S4 & $61(34)$ & $60(11)$ & $65(69)$ & $51(42)$ & $87(43)$ & $>95(49)$ & $66(69)$ \\
S5 & $60(10)$ & no conv. & $>95(86)$ & $81(31)$ & $85(22)$ & no conv. & $56(58)$ \\
\hline
\end{tabular}

General conditions: 2 equiv. TBHP $\left(5.5 \mathrm{M}\right.$ in decane), $50{ }^{\circ} \mathrm{C}, \mathrm{CHCl}_{3}, 0.001 \mathrm{~mol} \%$ precatalyst loading; conversion of substrate (\%) after $24 \mathrm{~h}$, as determined by GC-MS (selectivity in \% for epoxide in brackets); no conv. = substrate was not converted; unsel.= substrate was converted, but epoxide was not formed or consumed over reaction time; ${ }^{[a]}$ after $8 \mathrm{~h}$ reaction time; ${ }^{[b]}$ after $4 \mathrm{~h}$ reaction time; 
Table 4. Overview of conversion of substrate (selectivity to epoxide) with $\mathrm{H}_{2} \mathrm{O}_{2}$ as oxidant.

\begin{tabular}{ccccc} 
& $\mathbf{1}$ & $\mathbf{3}$ & $\mathbf{4}$ & $\mathbf{5}$ \\
\hline S1 & $32(>95)$ & $74(87)$ & $89(95)$ & $87(93)$ \\
S2 & no conv. & $30(77)$ & no conv. & $28(93)$ \\
S3 & no conv. & no conv. & no conv. & no conv. \\
S4 & no conv. & $64(27)$ & $39(31)$ & $65(10)$ \\
S5 & no conv. & unsel. & no conv. & unsel.
\end{tabular}

General conditions: 2 equiv. $\mathrm{H}_{2} \mathrm{O}_{2}\left(30 \mathrm{wt} \%\right.$ in water), $50{ }^{\circ} \mathrm{C}, \mathrm{CHCl}_{3}, 0.1 \mathrm{~mol} \%$ precatalyst loading; conversion of substrate (\%) after $24 \mathrm{~h}$, as determined by GC-MS (selectivity in \% for epoxide in brackets); no conv. = substrate was not converted; unsel.= substrate was converted, but no epoxide was formed or consumed over reaction time; $\mathbf{2 , 6}$ and $\mathbf{7}$ were unproductive/unselective, results not shown.

Table 5. Overview of TONs for epoxidations using TBHP/ $\mathrm{H}_{2} \mathrm{O}_{2}$.

\begin{tabular}{cccccccc}
\hline & $\mathbf{1}$ & $\mathbf{2}$ & $\mathbf{3}$ & $\mathbf{4}$ & $\mathbf{5}$ & $\mathbf{6}$ & $\mathbf{7}$ \\
\hline S1 & $75,500 / 270$ & $36,700 / 0$ & $95,300 / 650$ & $66,400 / 830$ & $68,400 / 790$ & $77,600 / 0$ & $110,000 / 0$ \\
S2 & $0 / 0$ & $0 / 0$ & $23,100 / 230$ & $0 / 0$ & $0 / 200$ & $0 / 0$ & $32,400 / 0$ \\
S3 & $0 / 0$ & $4,500 / 0$ & $15,000 / 0$ & $0 / 0$ & $0 / 0$ & $28,400 / 0$ & $27,200 / 0$ \\
S4 & $46,400 / 0$ & $6,800 / 0$ & $44,700 / 270$ & $21,500 / 120$ & $37,600 / 60$ & $48,000 / 0$ & $66,300 / 0$ \\
S5 & $29,500 / 0$ & $0 / 0$ & $83,500 / 0$ & $25,000 / 0$ & $18,600 / 0$ & $0 / 0$ & $56,200 / 0$ \\
\hline
\end{tabular}

TON is calculated per complex molecule.

In accordance to literature, also precatalysts 1-7 show higher epoxidation activity with organic oxidant TBHP compared to aqueous oxidant $\mathrm{H}_{2} \mathrm{O}_{2}$. Whilst conversion of cyclooctene $\mathbf{S} 1$ and selectivity for epoxide with TBHP was high in all cases, the more challenging substrates S2-5 showed a different picture. Only two (3 and 7 ) out of the seven complexes showed activity for 1octene $\mathbf{S 2}$, albeit with low conversions. For styrene $\mathbf{S 3}$ epoxidations suffered from un-selectivity $(0-34 \%)$ due to over-oxidation of the initially formed epoxide to phenylacetaldehyde and 
benzaldehyde. Epoxidation of cyclic substrate limonene S4 was selective for the endocyclic double bond, but selectivity overall (11-69\%) suffered from subsequent over-oxidation of the formed epoxide to the cyclohexanone carvone. An almost similar catalytic profile was observed for hydroxy containing substrate $\alpha$-terpineol S5, with the exception of precatalyst 3. For precatalysts 1, 4, 5 and 7, selectivity for epoxide with $\mathbf{S 5}$ was low (10-58\%) due to overoxidation, whereas mononuclear complexes $\mathbf{2}$ and $\mathbf{6}$ showed no conversion with $\mathbf{S 5}$ at all. Only precatalyst 3 both displayed high conversion (>95\%) and selectivity (86\%) (Table 3). In summary, dinuclear complexes $\mathbf{1}, \mathbf{3}, \mathbf{5}$ and $\mathbf{7}$ were in all cases more or at least similarly active (per molecule complex) than mononuclear precatalysts $\mathbf{2 , 4}$ and $\mathbf{6}$. This could, in part, be caused by a dinucleation in presence of adventitious water, resulting in a lowering of the precatalyst loading (per molecule).

Some of the trends observed in epoxidation with TBHP are also valid for epoxidations with $\mathrm{H}_{2} \mathrm{O}_{2}$, although the overall catalytic activity of precatalysts 1-7 was greatly reduced (Table 4 and Table 5). Again complex $\mathbf{3}$, in this case together with complex $\mathbf{5}$, showed the best performances with $\mathrm{H}_{2} \mathrm{O}_{2}$, both displaying catalytic activity with four (S1-2 and S4-5) out of the five substrates. Mononuclear complexes $\mathbf{2}$ and $\mathbf{6}$ showed no conversion with any substrate, making these two complexes the least active precatalysts out of the seven complexes. In contrast to TBHP epoxidations, also complex 7 remained unproductive in epoxidations of S1-4 and unselective for S5. This lack of catalytic activity with the aqueous oxidant $\mathrm{H}_{2} \mathrm{O}_{2}$ might be attributed to the enhanced water sensitivity of 7. Interestingly all seven complexes failed to give any conversion with aromatic substrate styrene $\mathbf{S 3}$ when $\mathrm{H}_{2} \mathrm{O}_{2}$ was the oxidant (

Table 4). In order to rule out a catalase-like activity of precatalysts $\mathbf{1 - 7}$, test reactions under catalytic conditions without added substrate were conducted. The concentration of $\mathrm{H}_{2} \mathrm{O}_{2}$ was 
determined after $24 \mathrm{~h}$ via iodometric titration, showing that complexes 1-7 do not decompose $\mathrm{H}_{2} \mathrm{O}_{2}$ under catalytic conditions.

Comparing the TONs (Table 5) allowed the conclusion that dinuclear complexes 3 and 7 exhibited the best overall catalytic activity, being the only two precatalysts showing activity for all five substrates S1-5 with TBHP as oxidant. Furthermore $\mathbf{3}$ and 7, bearing tert-butyl and chloride substituents at the phenolate moieties, respectively, gave the highest TONs for S1, suggesting a limited influence of the substituents electronic properties on the catalytic activity. However, the increased sensitivity of complex 7 can be attributed to the electron withdrawing nature of the chloride substituents, resulting in a weaker phenolate-Mo bond. From a comparison of the catalytic activities of complexes $\mathbf{3}$ and $\mathbf{5}$, it is evident that the tert-butyl substituted dinuclear compound $\mathbf{3}$ exhibits a better performance over a broader scope of substrates. Whereas this can partly be attributed to the low solubility of complex $\mathbf{5}$, it is also possible that the more acidic phenyl-amide moiety is prone to side-reactions such as deprotonation.

For complex 7 the precatalyst loading for $\mathbf{S} 1$ could be even reduced to $0.0005 \mathrm{~mol} \%$ (5 ppm), giving 54\% yield of cyclooctene oxide after $24 \mathrm{~h}$. At $0.001 \mathrm{~mol} \%$ loading, full conversion of S1 was obtained within $24 \mathrm{~h}$, indicating a $5 \mathrm{ppm}$ loading of 7 to be the limit of activity. Complex 3 on the other hand showed a remarkable activity with S5, hinting towards a high hydroxy functional group tolerance. Also complex 3 showed activity for three out of the five substrates with $\mathrm{H}_{2} \mathrm{O}_{2}$ as oxidant (Table 4). Taking into account the observed catalytic activities as well as the complexes stability and solubility, precatalysts $\mathbf{3}$ exhibits the best overall performance.

For these reasons, 3 and 7 were selected for further epoxidation experiments with cyclohexene substrates S6-S9 (Fig. 4). 


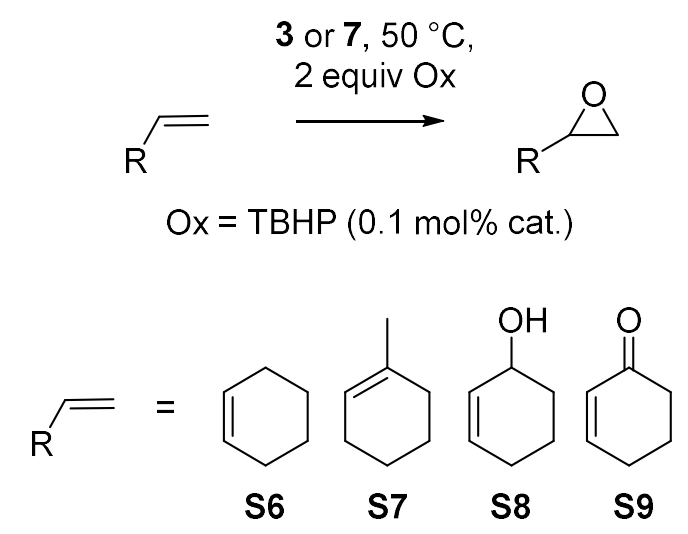

Fig. 4. Epoxidations of olefinic substrates S6-S9 by complexes 3 and 7

Table 6. Overview of conversion (selectivity to epoxide) of substrates S6-S9.

\begin{tabular}{ccc}
\hline & $\mathbf{3}$ & $\mathbf{7}$ \\
\hline S6 & $>95(>95)$ & $>95(>95)$ \\
S7 & $>95(>95)^{[\mathrm{a}]}$ & $>95(>95)^{[\mathrm{a}]}$ \\
S8 & $83(54)$ & $92(55)$ \\
S9 & no conv. & no conv.
\end{tabular}

General conditions: 2 equiv. TBHP (5.5 M in decane), $50{ }^{\circ} \mathrm{C}, \mathrm{CHCl}_{3}, 0.1 \mathrm{~mol} \%$ precatalyst loading; conversion of substrate (\%) after $24 \mathrm{~h}$, as determined by GC-MS (selectivity in \% for epoxide in brackets); no conv. $=$ substrate was not converted; ${ }^{[a]}$ conversion after $2 \mathrm{~h}$.

Similar to cyclic olefin S1, precatalysts 3 and 7 showed high activity and selectivity for substrates cyclohexene S6 and 1-methyl-1-cylohexene S7 (Table 6). Whereas especially complex $\mathbf{3}$ was very tolerant towards the hydroxy group in $\mathbf{S 5}$, in epoxidation of 2-cyclohexen-1-ol $\mathbf{S 8}$ both $\mathbf{3}$ and 7 were unselective, oxidizing $\mathbf{S 8}$ to 2-cyclohexen-1-one (S9) as the main side product. For S9 on the other hand, no activity was observed. To further test the tolerance for $\mathrm{OH}$ groups, complex $\mathbf{3}$ and $\mathbf{7}$ were used for the epoxidation of $\mathbf{S 1}$ in ethanol as solvent with TBHP as well as $\mathrm{H}_{2} \mathrm{O}_{2}$ as oxidant. Whereas 7 showed no activity in ethanol, 3 gave conversion to epoxide with both oxidants ( 
Table 7). Encouraged by this result we tested 3 in the four different alcohols methanol, ethanol, isopropanol and tert-butanol.

Table 7. Overview of conversion of substrate S1 (selectivity to epoxide) in alcoholic solvents

\begin{tabular}{cccccc}
\hline entry & precatalyst & loading [mol\%] & oxidant & solvent & conversion $^{[\mathrm{a}]}[\%]$ \\
\hline 1 & $\mathbf{3}$ & 1 & $\mathrm{H}_{2} \mathrm{O}_{2}$ & $\mathrm{EtOH}$ & $49(>95)$ \\
2 & $\mathbf{3}$ & 1 & TBHP & $\mathrm{EtOH}$ & $75(90)$ \\
3 & $\mathbf{3}$ & 0.1 & TBHP & $\mathrm{MeOH}$ & $63(>95)$ \\
4 & $\mathbf{3}$ & 0.1 & TBHP & $\mathrm{EtOH}$ & $59(93)$ \\
5 & $\mathbf{3}$ & 0.1 & TBHP & $i \mathrm{PrOH}$ & $75(90)$ \\
6 & $\mathbf{3}$ & 0.1 & TBHP & $t \mathrm{BuOH}$ & $89(95)$
\end{tabular}

General conditions: 2 equiv. oxidant, $50{ }^{\circ} \mathrm{C}$; ${ }^{[a]}$ conversion of substrate $(\%)$ after $24 \mathrm{~h}$, as determined by GC-MS (selectivity in \% for epoxide in brackets).

Precatalyst $\mathbf{3}$ is active for the epoxidation of substrate $\mathbf{S 1}$ in all four tested alcoholic solvents (

Table 7). With the aqueous oxidant $\mathrm{H}_{2} \mathrm{O}_{2}$, precatalyst activity was lower than compared to TBHP under the same reaction conditions (entry 1 and 2). In contrast to the standard solvent $\mathrm{CHCl}_{3}$, a single phase is formed in the reaction mixture, indicating that the lower activity with $\mathrm{H}_{2} \mathrm{O}_{2}$ cannot be solely attributed to a miscibility problem between precatalyst, oxidant and substrate. The activity of $\mathbf{3}$ in $\mathrm{MeOH}$ and $\mathrm{EtOH}$ is essentially the same (within experimental error), with conversions of 63 and 59\% respectively, for $\mathbf{S 1}$ (Table 7, entry 3 and 4). We could demonstrate that complex 4 is formed upon dissolution of $\mathbf{3}$ in methanol (Scheme 4). Based on the similar pKa value of EtOH (29.8)[26] and $\mathrm{MeOH}(29.0)[26]$ the formation of an analogous ethoxido complex is possible, hence explaining the similar catalytic activity. However, an induction period was only observed employing methanol as solvent (Fig. S5). The lack of conversion during the first hour of catalysis indicates the formation of the methoxido species 4 prior to 
formation of the catalytic active species. Epoxidations in isopropanol and tert-butanol show higher catalytic activity (75 and $89 \%$ conversion of S1, entry 5 and 6), despite the fact that tertbutanol is known to be an inhibitory by-product in epoxidations with TBHP as oxidant.[18,28] Mononuclear methoxido complexes 2, 4 and $\mathbf{6}$ have proven to be less active in epoxidation compared to their dinuclear congeners $\mathbf{1 ,}, \mathbf{3}, \mathbf{5}$ and $\mathbf{7}$, suggesting that the dinuclear complexes are stable in isopropanol or tert-butanol and are not cleaved to the corresponding mononuclear alkoxido complexes (vide supra).

Significant differences in catalytic activity of dinuclear compared to mononuclear precatalysts suggest a difference in the active catalyst species formed. We have no spectroscopic evidence for a cleavage of the dinuclear compounds in $\mathrm{CHCl}_{3}$, the standard solvent used in epoxidations. Thus, in presence of 10 equiv. TBHP dinuclear complex 3 remains unchanged as shown by ${ }^{1} \mathrm{H}$ NMR spectroscopy, indicating high stability of the complex under oxidative conditions (vide supra, Fig. S3). Based on this experimental evidence retention of the dinuclear structure during catalysis is suggested and thus also a different catalytically active species for mononuclear and dinuclear complexes. Furthermore, the high activity in tert-butanol is especially surprising, as tert-butanol usually impedes epoxidation activity with many other published precatalysts.[18,28] In comparison with structurally related, previously reported $\mu$-oxido bridged complexes (bearing hydrogen bond acceptor functionalities such as $-\mathrm{OMe}$ and $\left.-\mathrm{NMe}_{2}\right),[18]$ the high activity and functional group tolerance of the complexes herein clearly suggest a beneficial role of the additional hydrogen bond donors on the ligand amide functionalities, which seems to be able to stabilize the oxidant and/or substrate molecules in the second coordination sphere. Another benefit of the presented system might be the increased lability of the donor arm, allowing for the 
formation of a vacant coordination site. It remains unclear however, whether both molybdenum centers in dinuclear complexes $\mathbf{1 , 3 , 5}$ and $\mathbf{7}$ are active in epoxidation catalysis or not.

\section{Conclusions}

The synthesis of four novel aminophenolate ligands HL1-HL4 with tert-butyl and phenyl amide functionalities as hydrogen bond donors is reported. Upon reaction with the molybdenum(VI) precursor $\left[\mathrm{MoO}_{2}(\mathrm{acac})_{2}\right]$, they form, dependent on the used solvent, mono- and dinuclear complexes 1-7 of the general formulas $\left[\left\{\mathrm{MoO}_{2} \mathbf{L}\right\}_{2}(\mu-\mathrm{O})\right]$ and $\left[\mathrm{MoO}_{2} \mathbf{L}(\mathrm{OMe})\right]$, respectively, coordinated by one facially, tridentate ONO-ligand moiety per metal center.

Especially dinuclear complexes $\mathbf{1}, \mathbf{3}, \mathbf{5}$ and $\mathbf{7}$ were found to be highly efficient and stable precatalysts in the epoxidation of various olefins, leading to turnover numbers up to 110000 with precatalysts loadings of 5-10 ppm, although a lack of chemoselectivity due to over-oxidation was observed for some substrates. The complexes furthermore showed a remarkable tolerance towards hydroxy functionalities as well as moisture, allowing for the use of aqueous $\mathrm{H}_{2} \mathrm{O}_{2}$ as oxidant as well as alcoholic solvents such as EtOH and thus environmentally benign, "green", reaction conditions in catalysis. Furthermore also catalytic experiments in $t \mathrm{BuOH}$ (a by-product in epoxidations with TBHP, usually postulated to inhibit the reaction[18,28]) led to high epoxide yields with TBHP $(0.1 \mathrm{~mol} \%$ precatalyst $)$. The high activity and tolerance of the complexes is remarkable in comparison with previously reported, structurally similar compounds with acceptor functionalities (-OMe and $-\mathrm{NMe}_{2}$ ), which exhibited negligible activity in alcoholic solvents and/or with $\mathrm{H}_{2} \mathrm{O}_{2}$ as oxidant. Furthermore the system described herein allowed for the reduction of the precatalyst loading for all tested substrates by at least a factor of 10.[18] These findings underline the significant benefit of the introduction of hydrogen bond donor 
functionalities in epoxidation catalysis and therefore clearly warrant a further exploration and implementation in systems for efficient and "green" homogeneous epoxidation catalysis.

\section{Acknowledgements}

Financial support by the Austrian Science Fund (FWF, grant number P26264) and NAWI Graz is gratefully acknowledged.

\section{References}

[1] J. M. Brégeault J. Chem. Soc., Dalton Trans. (2003) 3289-3302.

[2] a) F. Cavani, J. H. Teles ChemSusChem 2 (2009) 508-534; b) T. Endo, A. Sudo J. Polym. Sci. A Polym. Chem. 47 (2009) 4847-4858.

[3] S. Huber, M. Cokoja, F. E. Kühn J. Organomet. Chem. 751 (2014) 25-32.

[4] A. K. Yudin, Aziridines and epoxides in organic synthesis, Wiley-VCH, Weinheim 2006.

[5] H. Adolfsson, D. Bellus, C. J. Forsyth, J. Houben, T. Weyl, Ethers, Thieme, Stuttgart 2008.

[6] a) Q.-H. Xia, H.-Q. Ge, C.-P. Ye, Z.-M. Liu, K.-X. Su Chem. Rev. 105 (2005) 1603-1662;

b) B. S. Lane, K. Burgess Chem. Rev. 103 (2003) 2457-2474; c) K. C. Gupta, A. K. Sutar Coord. Chem. Rev. 252 (2008) 1420-1450; d) H. Srour, P. Le Maux, S. Chevance, G.

Simonneaux Coord. Chem. Rev. 257 (2013) 3030-3050; e) J.-E. Bäckvall (Ed.) Modern Oxidation Methods, Wiley-VCH Verlag GmbH \& Co. KGaA, Weinheim, Germany 2010; f) K. A. Jørgensen Chem. Rev. 89 (1989) 431-458; g) H. Adolfsson, D. Balan in Aziridines and Epoxides in Organic Synthesis (Ed.: A. K. Yudin), Wiley-VCH Verlag GmbH \& Co. KGaA, Weinheim, FRG 2006.

[7] M. G. Clerici, M. Ricci, G. Strukul in Metal-catalysis in Industrial Organic Processes (Eds.: G. P. Chiusoli, P. M. Maitlis), Royal Society of Chemistry, Cambridge 2006. 
[8] R. M. Bullock, Catalysis without precious metals, Wiley-VCH, Weinheim 2010.

[9] L. M. Peschel, J. A. Schachner, F. Belaj, N. C. Mösch-Zanetti Eur. J. Inorg. Chem. (2017) $2808-2817$.

[10] a) M. Bagherzadeh, L. Tahsini, R. Latifi, L. K. Woo Inorg. Chim. Acta 362 (2009) 36983702; b) F. E. Kühn, A. M. Santos, M. Abrantes Chem. Rev. 106 (2006) 2455-2475; c) J. A. Schachner, P. Traar, C. Sala, M. Melcher, B. N. Harum, A. F. Sax, M. Volpe, F. Belaj, N. C. Mösch-Zanetti Inorg. Chem. 51 (2012) 7642-7649; d) J. Pisk, B. Prugovečki, D. MatkovićČalogović, R. Poli, D. Agustin, V. Vrdoljak Polyhedron 33 (2012) 441-449; e) J. Pisk, D. Agustin, V. Vrdoljak, R. Poli Adv. Synth. Catal. 353 (2011) 2910-2914; f) J. Morlot, N. Uyttebroeck, D. Agustin, R. Poli ChemCatChem 5 (2013) 601-611; g) I. Sheikhshoaie, A. Rezaeifard, N. Monadi, S. Kaafi Polyhedron 28 (2009) 733-738; h) A. Rezaeifard, I. Sheikhshoaie, N. Monadi, M. Alipour Polyhedron 29 (2010) 2703-2709; i) M. Bagherzadeh, R. Latifi, L. Tahsini, V. Amani, A. Ellern, L. K. Woo Polyhedron 28 (2009) 2517-2521; j) J. M. Sobczak, J. J. Ziółkowski Appl. Catal., A 248 (2003) 261-268; k) J. Zhao, X. Zhou, A. M. Santos, E. Herdtweck, C. C. Romão, F. E. Kühn Dalton Trans. (2003) 3736-3742; 1) Y. Sui, X. Zeng, X. Fang, X. Fu, Y. Xiao, L. Chen, M. Li, S. Cheng J. Mol. Catal. A: Chem. 270 (2007) 61-67.

[11] P. Traar, J. A. Schachner, B. Stanje, F. Belaj, N. C. Mösch-Zanetti J. Mol. Catal. A: Chem. 385 (2014) 54-60.

[12] J. W. Kück, R. M. Reich, F. E. Kühn Chem. Rec. 16 (2016) 349-364.

[13] a) C. Müller, N. Grover, M. Cokoja, F. E. Kühn in Advances in Inorganic Chemistry, Elsevier 2013; b) N. Grover, A. Pöthig, F. E. Kühn Catal. Sci. Technol. 4 (2014) 4219-4231; c) S. M. Bruno, S. S. Balula, A. A. Valente, F. A. Almeida Paz, M. Pillinger, C. Sousa, J. 
Klinowski, C. Freire, P. Ribeiro-Claro, I. S. Gonçalves J. Mol. Catal. A: Chem. 270 (2007) 185-194; d) M. Abrantes, A. M. Santos, J. Mink, F. E. Kühn, C. C. Romão Organometallics 22 (2003) 2112-2118; e) M. E. Judmaier, C. Holzer, M. Volpe, N. C. Mösch-Zanetti Inorg. Chem. 51 (2012) 9956-9966.

[14] a) M. G. Clerici, O. A. Kholdeeva (Eds.) Liquid Phase Oxidation via Heterogeneous Catalysis, John Wiley \& Sons, Inc, Hoboken, New Jersey 2013; b) G. Grigoropoulou, J. H. Clark, J. A. Elings Green Chem. 5 (2003) 1-7.

[15] a) N. Zwettler, M. E. Judmaier, L. Strohmeier, F. Belaj, N. C. Mösch-Zanetti Dalton Trans. 45 (2016) 14549-14560; b) A. Dupé, M. E. Judmaier, F. Belaj, K. Zangger, N. C. Mösch-Zanetti Dalton Trans. 44 (2015) 20514-20522; c) K. Most, S. Köpke, F.

Dall'Antonia, N. C. Mösch-Zanetti Chem. Commun. (2002) 1676-1677; d) G. Lyashenko, G. Saischek, M. E. Judmaier, M. Volpe, J. Baumgartner, F. Belaj, V. Jancik, R. HerbstIrmer, N. C. Mösch-Zanetti Dalton Trans. (2009) 5655-5665; e) G. Lyashenko, G. Saischek, A. Pal, R. Herbst-Irmer, N. C. Mösch-Zanetti Chem. Commun. (2007) 701-703; f) M. Volpe, N. C. Mösch-Zanetti Inorg. Chem. 51 (2012) 1440-1449; g) N. C. Mösch-Zanetti, D. Wurm, M. Volpe, G. Lyashenko, B. N. Harum, F. Belaj, J. Baumgartner Inorg. Chem. 49 (2010) 8914-8921; h) K. Most, J. Hoßbach, D. Vidovic, J. Magull, N. C. Mösch-Zanetti Adv. Synth. Catal. 347 (2005) 463-472; i) N. Zwettler, N. Grover, F. Belaj, K. Kirchner, N. C. Mösch-Zanetti Inorg. Chem. 56 (2017) 10147-10150.

[16] a) N. Zwettler, J. A. Schachner, F. Belaj, N. C. Mösch-Zanetti Inorg. Chem. 53 (2014) 12832-12840; b) N. Zwettler, J. A. Schachner, F. Belaj, N. C. Mösch-Zanetti Inorg. Chem. 55 (2016) 5973-5982; c) P. Traar, J. A. Schachner, L. Steiner, A. Sachse, M. Volpe, N. C. Mösch-Zanetti Inorg. Chem. 50 (2011) 1983-1990. 
[17] A. Dupé, M. K. Hossain, J. A. Schachner, F. Belaj, A. Lehtonen, E. Nordlander, N. C. Mösch-Zanetti Eur. J. Inorg. Chem. (2015) 3572-3579.

[18] M. E. Judmaier, C. H. Sala, F. Belaj, M. Volpe, N. C. Mösch-Zanetti New J. Chem. 37 (2013) 2139.

[19] M. E. Judmaier, C. Holzer, M. Volpe, N. C. Mösch-Zanetti Inorg. Chem. 51 (2012) 9956-9966.

[20] a) T. Taguchi, R. Gupta, B. Lassalle-Kaiser, D. W. Boyce, V. K. Yachandra, W. B. Tolman, J. Yano, M. P. Hendrich, A. S. Borovik J. Am. Chem. Soc. 134 (2012) 1996-1999; b) R. L. Shook, S. M. Peterson, J. Greaves, C. Moore, A. L. Rheingold, A. S. Borovik J. Am. Chem. Soc. 133 (2011) 5810-5817; c) C. E. MacBeth, A. P. Golombek, V. G. Young Jr., C. Yang, K. Kuczera, M. P. Hendrich, A. S. Borovik Science 289 (2000) 938-941; d) S. A. Cook, E. A. Hill, A. S. Borovik Biochemistry 54 (2015) 4167-4180; e) S. A. Cook, A. S. Borovik Acc. Chem. Res. 48 (2015) 2407-2414.

[21] N. Zwettler, A. Dupé, J. A. Schachner, F. Belaj, N. C. Mösch-Zanetti Inorg. Chem. 54 (2015) 11969-11976.

[22] a) S. A. Hauser, M. Cokoja, F. E. Kühn Catal. Sci. Technol. 3 (2013) 552; b) A. Schmidt, N. Grover, T. K. Zimmermann, L. Graser, M. Cokoja, A. Pöthig, F. E. Kühn J. Catal. 319 (2014) 119-126; c) D. Betz, A. Raith, M. Cokoja, F. E. Kühn ChemSusChem 3 (2010) 559562.

[23] a) F. P. Byrne, S. Jin, G. Paggiola, T. H. M. Petchey, J. H. Clark, T. J. Farmer, A. J. Hunt, C. Robert McElroy, J. Sherwood Sustain Chem Process 4 (2016) 1034; b) C. Capello, U. Fischer, K. Hungerbühler Green Chem. 9 (2007) 927.

[24] H. Gehrke, J. Veal Inorg. Chim. Acta 3 (1969) 623-627. 
[25] G. M. Sheldrick Acta Crystallogr., Sect. A: Found. Crystallogr. 64 (2008) 112-122.

[26] W. N. Olmstead, Z. Margolin, F. G. Bordwell J. Org. Chem. 45 (1980) 3295-3299.

[27] J. M. Mayer Inorg. Chem. 27 (1988) 3899-3903.

[28] S. Gago, P. Neves, B. Monteiro, M. Pessêgo, A. D. Lopes, A. A. Valente, F. A. Almeida Paz, M. Pillinger, J. Moreira, C. M. Silva et al. Eur. J. Inorg. Chem. (2009) 4528-4537. 